\title{
Extrasynaptic signaling enables an asymmetric juvenile motor circuit to produce a symmetric gait
}

\author{
Yangning Lu $u^{1,2, a}$, Tosif Ahamed ${ }^{2, a}$, Ben Mulcahy ${ }^{2}$, Daniel Witvliet ${ }^{2,3}$, Sihui Asuka Guan ${ }^{1,2}$, Douglas Holmyard ${ }^{2}$, Wesley Hung ${ }^{2}$, Jun \\ Meng $^{1,2}$, Quan Wen ${ }^{5,6}$, Andrew Chisholm ${ }^{7}$, Aravinthan DT Samuel ${ }^{5}$, and Mei Zhen ${ }^{1,2,3,4^{*}}$ \\ ${ }^{1}$ Department of Physiology, University of Toronto, Toronto, ON, Canada, ${ }^{2}$ Lunenfeld-Tanenbaum Research Institute, Mount Sinai Hospital, Toronto, ON, Canada, ${ }^{3}$ Department of \\ Molecular Genetics, University of Toronto, Toronto, ON, Canada, ${ }^{5}$ Department of Physics and Center for Brain Science, Harvard University, Cambridge, MA, USA, ${ }^{6}$ School of Life \\ Sciences, University of Science and Technology, Hefei, Anhui, China, ${ }^{7}$ Department of Biology, University of California, San Diego, CA, ${ }^{a}$ These authors contributed equally, \\ *Correspondence: meizhen@lunenfeld.ca
}

\begin{abstract}
Bilaterians generate motor patterns with symmetries that correspond to their body plans. This is thought to arise from wiring symmetries in their motor circuits. We show that juvenile C. elegans larva has an asymmetrically wired motor circuit, but still generates a bending pattern with dorsal-ventral symmetry. In the juvenile circuit, wiring between excitatory and inhibitory motor neurons coordinates contraction of dorsal muscles with relaxation of ventral muscles, producing dorsal bends. Ventral bending is not driven by analogous wiring. Instead, ventral muscles are excited uniformly by premotor interneurons through extrasynaptic signaling. Ventral bends occur in anti-phasic entrainment to activity of the same motor neurons that drive dorsal bends. During maturation, the juvenile motor circuit is replaced by two homologous motor circuits that separately drive dorsal and ventral bending. Modeling reveals that the juvenile's immature motor circuit provides an adequate solution to generate an adult-like gait long before the animal matures. Circuit degeneracy minimizes functional disruption during development.
\end{abstract}

\section{Introduction}

Symmetry in animal gaits is thought to mirror underlying symmetries in motor circuits and body plans. In vertebrates, hind-limb movements are driven by rhythmogenic spinal interneuron circuits called half-centers ${ }^{1,2}$. Structurally symmetric half-centers coordinate contraction and relaxation of muscle groups in each limb. Phase relations between half-centers for left and right limbs produce alternating gaits $^{3-5}$.

C. elegans generates alternating dorsal-ventral bending waves for locomotion ${ }^{6}$. The wiring diagram of its adult motor circuit contains symmetric inputs onto dorsal and ventral body wall muscles. This motor system consists of two homologous motor neuron networks ${ }^{7,8}$, analogous to symmetric half-centers. In this circuit, both dorsal and ventral muscles form neuromuscular junctions (NMJs) with cholinergic excitatory motor neurons (eMNs) that contract muscles, as well as with GABAergic inhibitory motor neurons (iMNs) that relax muscles $^{9-11}$

One motor subcircuit consists of repeated modules of cholinergic eMNs (DA and DB), and GABAergic iMNs (VD). They coordinate simultaneous dorsal muscle contraction and ventral muscle relaxation in each body segment, producing dorsal bends. A complementary motor subcircuit produces ventral bends, with symmetric wiring of a homologous set of eMNs (VA and VB) and iMNs (DD) ${ }^{8}$. Alternating activity of these two subcircuits in each body segment gives rise to alternating dorsal and ventral bending during adult locomotion $^{12}$.

However, the newly born larva (L1) lacks this wiring symmetry (Fig. 1A, B). For the first few hours after birth, an L1 larva has only DA, DB, and DD motor neurons ${ }^{13}$. A partial serial section electron microscopy (ssEM) reconstruction of the L1 larva ${ }^{7}$ suggests that its motor neuron wiring is different from that in the adult ${ }^{8}$. In examined larva body segment, eMNs (DA and DB) make NMJs exclusively to dorsal muscles and iMNs (DD) make NMJs exclusively to ventral muscles. NMJs from eMNs are also dyadic synapses to the iMNs. Hence the entire L1 motor circuit resembles the adult's subcircuit for dorsal bending. However, despite lacking a homologous circuitry to drive ventral bends, the L1 larva crawls with an adult-like gait.

Here, we sought mechanisms by which a structurally asymmetric motor circuit produces a symmetric gait. First, we used ssEM to fully reconstruct the connectivity between premotor interneurons, motor neurons, and muscle cells in the L1 motor circuit. We then used functional imaging, optogenetic perturbations, as well as synapse and cell ablation to assess the role of each circuit component in generating dorsal-ventral bends. Finally, we combined modeling and behavioral simulations to develop an integrated understanding of underlying mechanisms.

We found that the L1 motor circuit is networked by both synaptic and extrasynaptic connections. Its motor neurons form a self-regulated circuit to generate and exit dorsal bends. Ventral bends are produced by anti-phasic entrainment: extrasynaptic transmission from cholinergic premotor interneurons (eINs) excites ventral muscles uniformly along the body, which allows the same motor neurons for dorsal bending to produce complementary ventral bends.

We present here an example of circuit degeneracy ${ }^{14}$ that minimizes functional disruption during development, and an alternative solution that generates a symmetric gait from an asymmetrically wired motor circuit. With extrasynaptic transmission and neurons adopting additional roles, an immature motor circuit can generate a mature gait. These are adaptive strategies by which an animal maintains behavioral output despite substantial structural changes.

\section{Results}

The L1 motor circuit has asymmetric wiring to dorsal and ventral body wall muscles

C. elegans is born with a fraction of motor neurons of the adult motor circuit. Beginning at the mid-L1 larva stage, post-embryonic neurogenesis gives rise to new motor neurons ${ }^{15}$. Because post-embryonic neurons contain all that innervate ventral body wall muscles in the adults ${ }^{8}$, the L1 larva must have a distinct, and potentially asymmetrically wired motor circuit. 
A

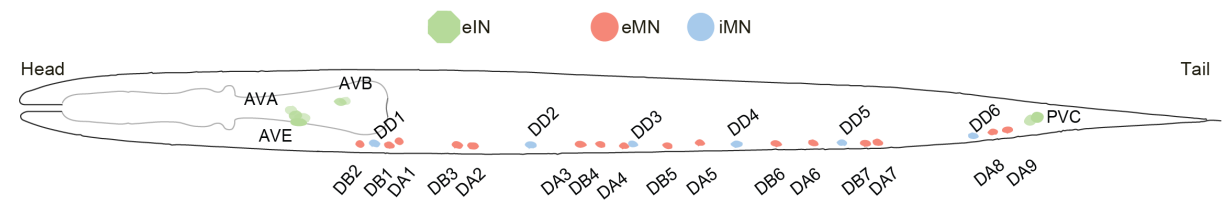

B

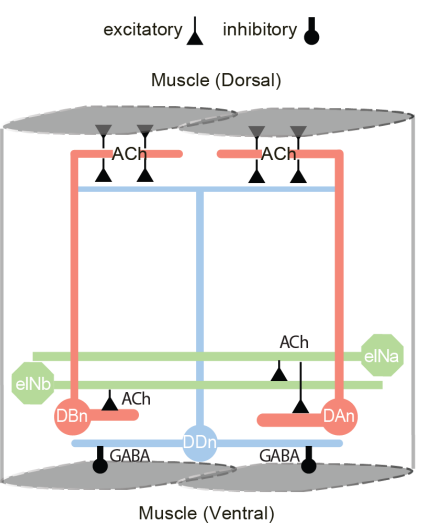

C

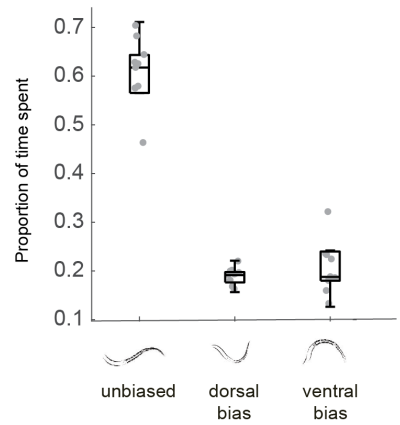

D

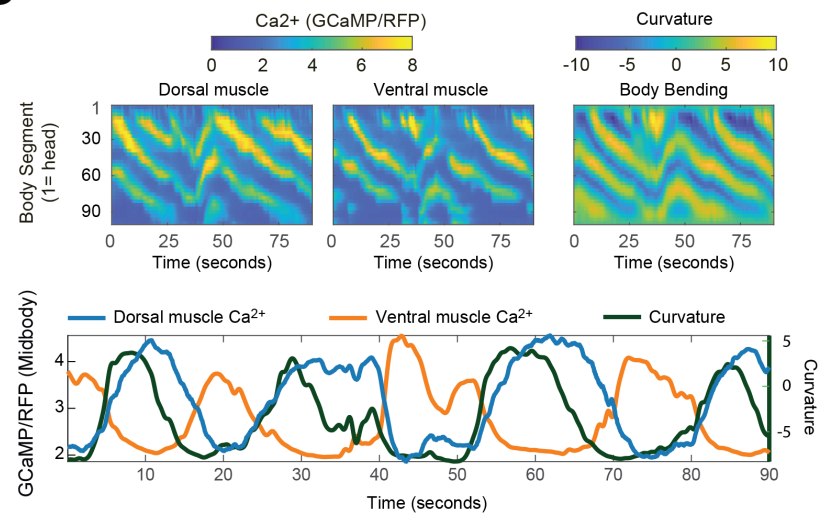

$\mathbf{E}$

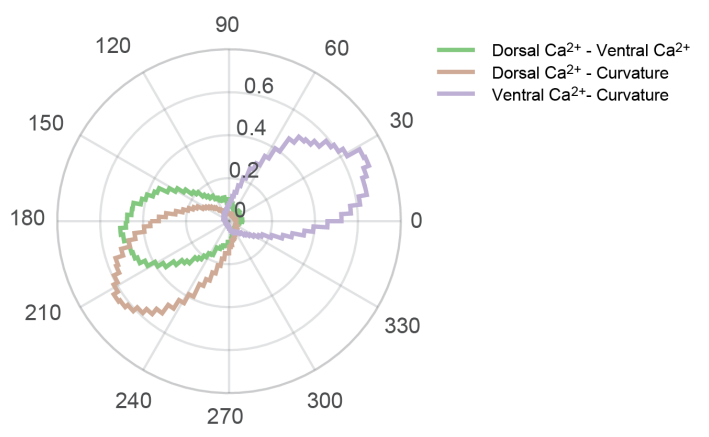

Figure 1. L1 larvae exhibit asymmetry in motor circuit wiring but symmetry in gait pattern and muscle activity. A. Schematic of the motor circuit for body movement in a newborn C. elegans L1 larva. Circles denote neuron soma, color-coded by class. B. Schematic of their synaptic wiring, deduced by serial section transmission EM reconstruction (ACh: Acetylcholine). C. L1 larvae swim without a dorsal-ventral bias most of the time and spend roughly equal time in postures with a dorsal or ventral bias. D. Calcium dynamics of dorsal and ventral muscles in a crawling L1 larva. Colormap represent the GCaMP3/RFP ratio in muscles (top left panels) and the body curvature (top right panel), respectively. (lower panel) Time series of curvature, dorsal and ventral muscle activity at mid-body (segment 50). E. Polar histograms of the phase difference between curvature, dorsal muscle activity, and ventral muscle activity in the body (segments 33-95). $n=12$ larvae.

We used serial EM to fully reconstruct the motor circuit of multiple L1 larvae, from premotor interneurons to motor neurons to muscles (Mulcahy et al., in preparation; this study). In the mid-L1 larva (Methods; Fig. 1; Fig. S1), for motor neurons, we confirmed results from a previous partial reconstruction ${ }^{7}$ : inputs to dorsal and ventral muscles are fundamentally asymmetric (Fig. 1B). All cholinergic eMNs (DA and DB) make NMJs only to dorsal muscles. All GABAergic iMNs (DD) make NMJs only to ventral muscles. Most NMJs from the eMNs are dyadic, innervating dorsal muscles as well as the iMNs that project to the opposite side (examples in Fig. S1). Multiple eMNs and iMNs form a chain of similarly wired modules along the body (illustrated in Fig. 1A, B).

In the adult motor circuit, two different groups of premotor interneurons (eINs) innervate the A- and B-class eMNs to regulate directional movements ${ }^{12,16}$. Our EM reconstruction revealed that this wiring pattern is already present in the newborn L1 larva (Fig. 1B; Fig S1). The DA subclass of eMNs are postsynaptic to cholinergic eINs that regulate backward locomotion (AVA, AVE, AVD); the DB subclass eMNs are postsynaptic to eINs that promote forward locomotion (AVB and PVC). Thus the key difference in structural wiring between the L1 and adult motor circuits is at the layer between motor neurons to muscles.

\section{L1 larvae generate alternating dorsal-ventral body bends and muscle activities}

Despite a dorsal-ventral wiring asymmetry, C. elegans's alternating dorsal-ventral bending pattern is established at birth. When swimming, L1 larvae exhibited sinusoidal bending waves and full-body coils without dorsal or ventral bias (Fig. 1C; Supplemental movie 1). When crawling, L1 larvae propagated alternating dorsal-ventral bending waves along the body (Fig. 1D; E; Supplemental movie 1).

Calcium dynamics of body wall muscles correlated with this bending pattern. In slowly crawling L1 larvae (Fig. S2; Supplemental movie 2), calcium waves propagated along dorsal and ventral muscles (Fig. 1D), and tracked curvature changes (Fig. 1E). Dorsal-ventral alternation in body bending correlated with out-of-phase activation of the corresponding muscles (Fig. 1E). Phase shift between the dorsal and ventral 
A

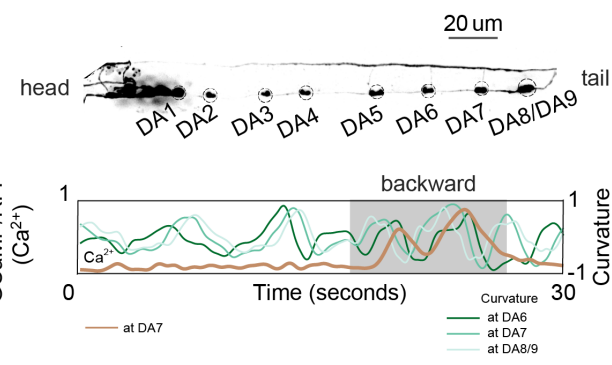

B
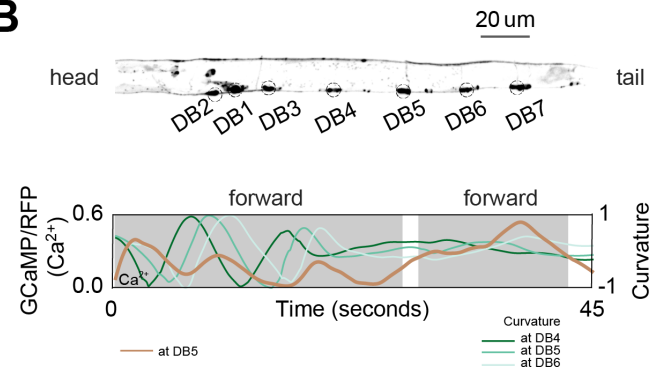

C

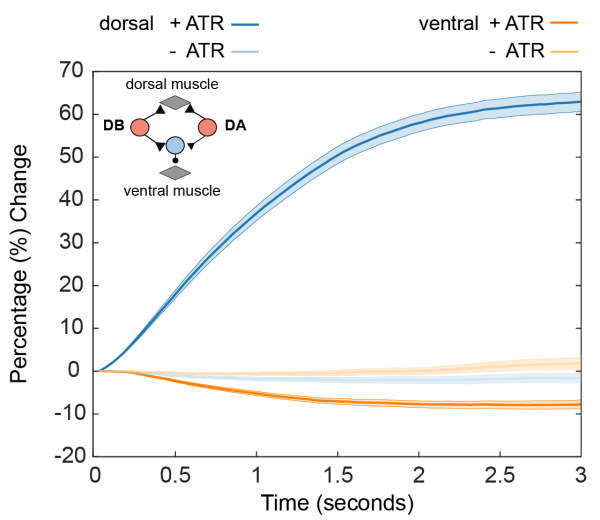

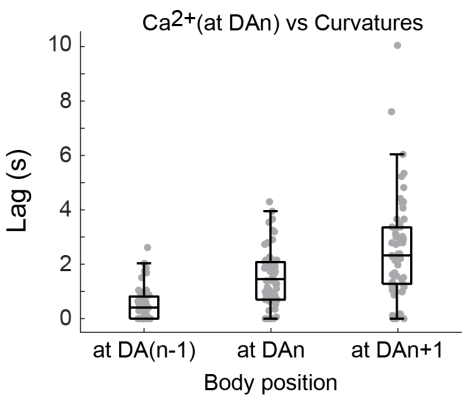

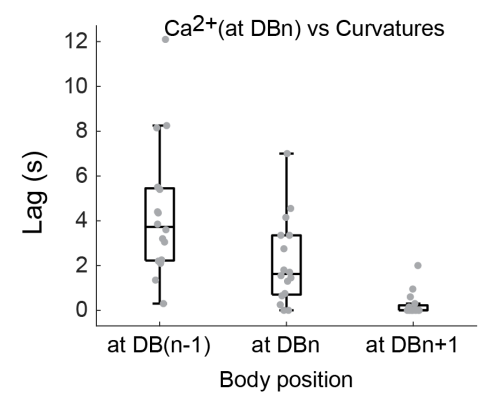

D

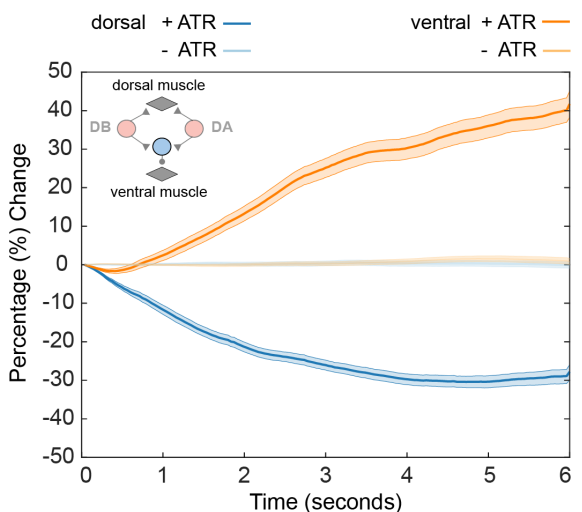

Figure 2. Cholinergic eMNs underlie dorsal muscle contraction. A. Left panel: (top) GCaMP6s expression in the DA subclass eMNs in an L1 larva. (bottom) An example trace of calcium activity for DA7 motor neuron (left axis), overlaid with curvatures at different body segments (right axis). Shaded area denotes backward movement. Right panel: Shortest time lag between calcium activity and curvature changes at regions anterior (N-1), at (N), and posterior ( $N+1)$ to the neuron's soma (DAn). B. Left panel: (top): GCaMP6s expression in the DB subclass eMNs. (bottom): an example calcium trace for DB5 motor neuron (left axis), overlaid with curvatures at different body segments (right axis). Right panel: Shortest time lag between calcium activity and curvature changes at body segments anterior $(\mathrm{N}-1)$, at $(\mathrm{N})$, and posterior $(\mathrm{N}+1)$ to the neuron's soma (DBn). C. Simultaneous activation of all eMNs by Chrimson and calcium imaging of body wall muscles in L1 larvae. Y-axis plots percentage changes of the muscle activity from $t=0$. Control group (-ATR): 21 stimulation epochs from 7 larvae. Experimental group: (+ATR): 33 stimulation epochs from 12 larvae. D. Simultaneous inactivation of eMNs by GtACR2 and calcium imaging of body wall muscles in L1 larvae. Control group (-ATR): 39 stimulation epochs from 8 larvae. Experimental group (+ATR): 35 stimulation epochs from 12 larvae. Lines and shades denote median and $95 \%$ confidence intervals, respectively.

muscle activity with bending (Fig. 1D, E) was dependent on crawling speed (Fig. S2). Overall dorsal and ventral muscle activity level is balanced.

\section{Cholinergic excitatory motor neurons drive dorsal muscle contraction}

Wiring asymmetry of the L1 motor circuit needs to be reconciled with bending symmetry of the L1 larva. One hypothesis is cholinergic and GABAergic synapses may have mixed signs (inhibitory or excitatory) in L1, compensating for the wiring asymmetry. To determine the functional relationship between cholinergic motor neurons and bending, we measured calcium dynamics of eMNs in moving L1 larvae.

We found that when L1 larvae moved backwards, DA motor neurons were activated in an anterograde sequence as bending traveled from tail to head (Fig. 2A; Supplemental movie 3). Calcium dynamics at each DA soma correlated with dorsal bending, exhibiting the shortest time lag with that of the anterior body segment (Fig. 2A, right panel). This position-dependent correlation is consistent with the anatomical wiring: DA axons project anteriorly, making NMJs to dorsal muscles anterior to their soma (Fig. 1B).

When L1 larvae moved forward, DB motor neurons were activated in a retrograde sequence as bending traveled from head to tail (Fig. 2B; Supplemental movie 4). Calcium dynamics at each DB soma correlated with dorsal bending, exhibiting the shortest time lag with that of the posterior body segment (Fig. 2B, right panel), consistent with the posterior projection of DB axons (Fig. 1B). Both results establish positive correlations between eMNs and dorsal bending.

To determine whether eMNs directly activate dorsal muscles, we imaged muscle calcium dynamics while simultaneously manipulating motor neuron activity (see Methods). We found that co-activation of all eMNs by 
A

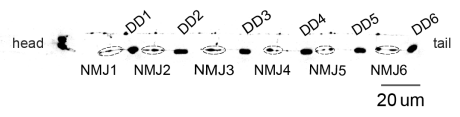

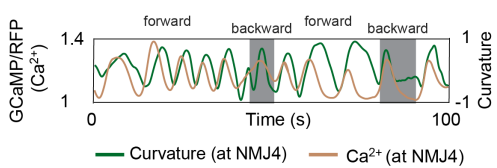

C

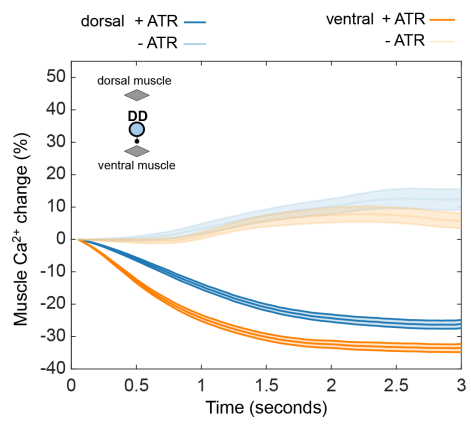

B
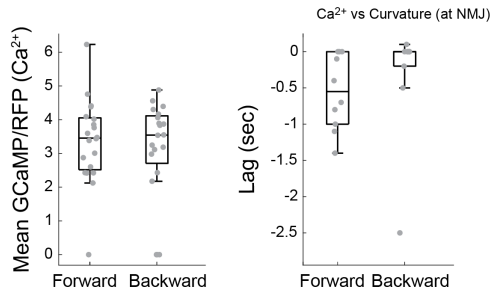

D

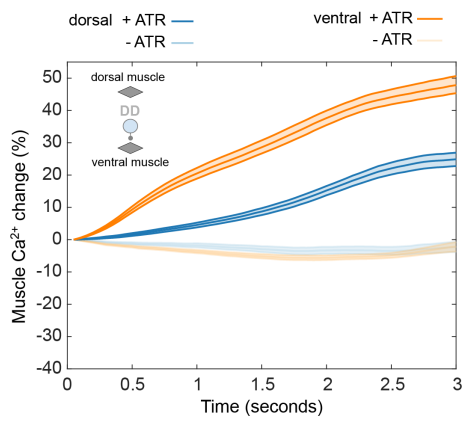

$\mathbf{E}$
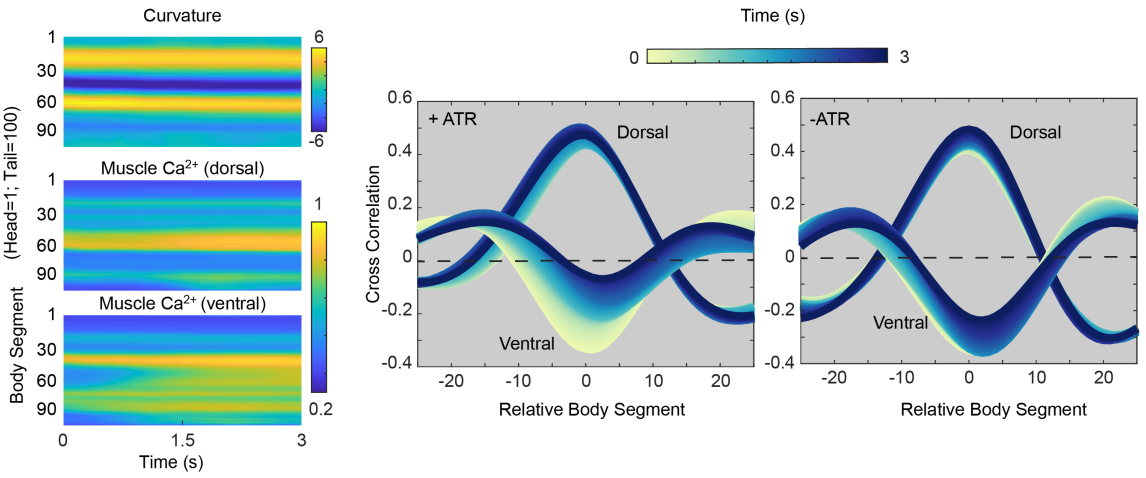

$\mathbf{F}$

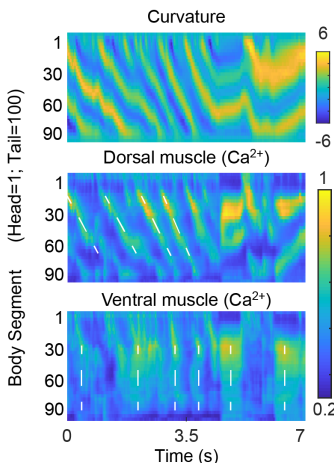

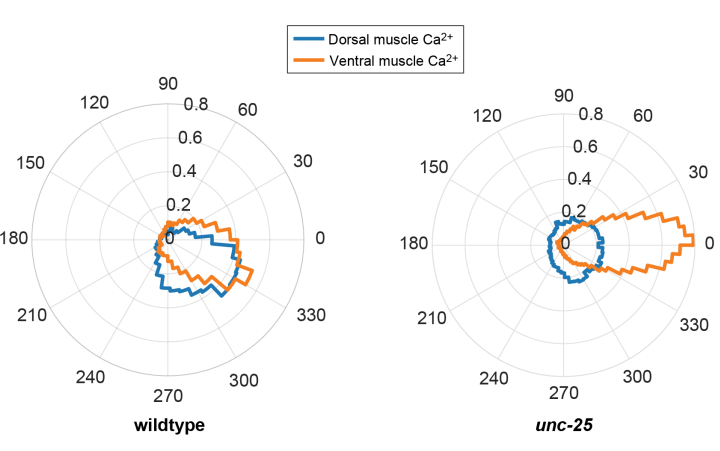

Figure 3. GABAergic iMNs underlie ventral and dorsal muscle relaxation A. (top) GCaMP6s expression in the DD motor neurons in an L1 larva. Each DD makes a tight cluster of NMJs; NMJn is from DDn. (bottom) An example calcium trace of NMJ4 (left axis), overlaid with body curvature at the same segment (right axis). Shaded areas denote periods of backward movements. B. (Left) Mean calcium activity of DD NMJs during forward and backward movement. $n=17$ NMJs from 12 larvae; (Right) Shortest time lags between calcium activity and curvature changes during forward and backward movements. C. Simultaneous GABAergic iMN (DD NMJs) Chrimson activation and muscle calcium imaging in L1 larvae. Y-axis plots percentage changes of the muscle activity from $t=0$. Control group (-ATR): $\mathrm{n}=13$ stimulation epochs from 7 larvae; Experimental group (+ATR): $\mathrm{n}=18$ stimulation epochs from 10 larvae. D. Simultaneous GABAergic iMN (DD) GtACR2 inhibition and muscle calcium imaging in L1 larvae. Control group (-ATR): $\mathrm{n}=$ 10 stimulation epochs from 5 larvae; Experimental group (+ATR): $n=19$ stimulation epochs from 10 larvae. Lines and shades in $C$ and $D$ represent median and $95 \%$ confidence interval, respectively. E.(Left) Example heatmap of curvature and dorsal and ventral muscle calcium of an L1 larva during iMN GtACR2 inactivation. (Right) Spatial cross-correlation between the muscle calcium and curvature along the body (segment 33-95) during the course of iMN inactivation. Color encodes the time-points at which the cross-correlation was calculated. Only for ventral muscles in the presence of ATR, the correlation decreased towards zero. Quantified from the same data as in D. F. (left) Example heatmap of curvature and dorsal and ventral muscle calcium in a crawling mutant larva that cannot synthesize GABA (unc-25). Dashed lines denote propagating calcium waves in dorsal muscles, and non-propagating waves in ventral muscles. (right) Polar histograms of muscle activity phase lags between segments along the body in wildtype and unc-25 mutant L1 larvae. The body (segments 33-95) is binned into 6 equally spaced sections. Phase differences are calculated between adjacent sections separately for dorsal and ventral muscles.

Chrimson ${ }^{17}$ increased the activity of dorsal muscles, but not ventral muscles (Fig. 2C). Similarly, inhibition of eMNs by
GtACR $2^{18}$ lowered the activity of dorsal muscles, but led to an activity increase in ventral muscles (Fig. 2D). Cholinergic 
inputs from eMNs are thus excitatory specifically to dorsal muscles.

\section{GABAergic inhibitory motor neurons promote ventral and dorsal muscle relaxation}

GABAergic motor neurons (DD) make NMJs exclusively to ventral body wall muscles. During early postnatal development, GABAergic synapses from rodent interneurons are not inhibitory but excitatory ${ }^{19}$. In $C$. elegans, whether GABAergic signaling promotes muscle relaxation in L1 larvae ${ }^{20}$, as in adults ${ }^{21}$, was unclear ${ }^{22}$.

To assess how DD motor neurons regulate muscle activity, we measured their dynamics in crawling L1 larvae. Each DD motor neuron makes a cluster of NMJs that can be recorded as a single region of ROI (Fig. 3A, NMJn). Whether these animals moved backward or forward, activity rise of DD NMJs correlated with ventral relaxation (Fig. 3B; Supplemental movie 5), and the calcium rise preceded decreased curvature at each ROI (Fig. 3B).

To determine whether DD motor neurons inhibit ventral muscles, we imaged muscle calcium dynamics while manipulating DD activity. As expected from inhibitory NMJs, activation of DD by Chrimson strongly reduced ventral muscle activity (Fig. 3C). Unexpectedly, their activation also led to a smaller reduction of dorsal muscle activity (Fig. 5B), even though DD motor neurons do not make NMJs to dorsal muscles (Fig. 3B).

Inhibition of DD motor neurons by GtACR2 had an opposite effect: a strong increase in ventral muscle activity, accompanied by a smaller increase in dorsal muscle activity (Fig. 3D; Supplemental movie 6). More intriguingly, activity increase of dorsal and ventral muscles exhibited spatial differences: while dorsal activity continued to track curvature, ventral activity increase was uniform along the body, decreasing correlations with curvature over time (Fig. 3E, Supplemental movie 6).

Spatial difference between dorsal and ventral muscle activity was also observed when we genetically silenced DD motor neurons. In crawling mutant larvae that do not synthesize GABA (GAD/unc-25; Supplemental movie 7), dorsal muscles exhibited calcium dynamics that correlated with propagating bending waves, whereas calcium signals of ventral muscles did not propagate, disrupting correlation with curvature (Fig. 3F; Fig. S3).

Therefore DD motor neurons are inhibitory at birth. These iMNs inhibit ventral muscles with synapses and inhibit dorsal muscles without direct synaptic input. Dorsal and ventral muscles exhibit structural, temporal and spatial differences in their relationships with iMNs, implicating distinct inhibitory mechanisms.

\section{Excitatory and inhibitory motor neurons form a circuit for dorsal bending}

Most NMJs from eMNs to dorsal muscles are dyadic synapses, juxtaposing dendrites of iMNs that inhibit the opposing ventral muscles (Fig. S1). Thus, activation of dorsal muscles should lead to contralateral ventral muscle inhibition.

Consistent with this hypothesis, activation of all eMNs by Chrimson increased the iMN activity (Fig. 4; Supplemental movie 8 a). Inhibition of all eMNs by GtACR2 decreased the

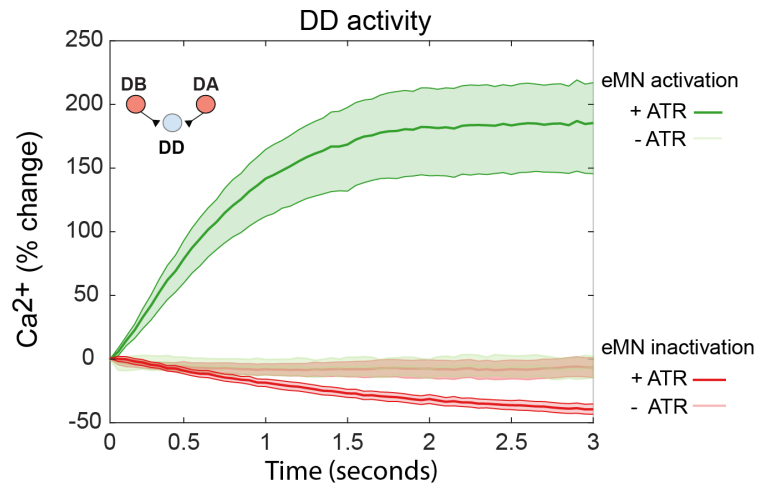

Figure 4. Optogenetic activation and inactivation of eMNs leads to corresponding changes in iMN activity. (inset) Schematic depicting the wiring between iMNs and eMNs. (green) Activation of eMNs by Chrimson elevates iMN activity. -ATR group: $n$ $=20$ stimulation epochs from 5 larvae; + ATR group: $n=23$ stimulation epochs from 9 larvae. (red) Inhibition of eMNs by Archaerhodopsin decreases DD activity. -ATR group: $\mathrm{n}=25$ stimulation epochs from 6 larvae; +ATR group: $\mathrm{n}=21$ stimulation epochs from 9 larvae. Lines and shades denote median and $95 \%$ confidence intervals, respectively.

iMN activity (Fig. 4; Supplemental movie 8b). Furthermore, ablation of the DB subclass eMNs by miniSOG ${ }^{23}$ preferentially reduced DD's activity when animals attempted to move forward, whereas ablation of the DA subclass eMNs reduced DD's activity when animals attempted to move backward (Fig. S4).

We have shown that NMJs to dorsal muscles and ventral muscles drive contraction and relaxation, respectively (Fig. 2; Fig. 3). Dyadic NMJs from eMNs to iMNs promote relaxation of juxtaposed ventral muscles when dorsal muscles contract. This coordination generates dorsal bending (Fig. 4).

\section{Extrasynaptic inhibition from GABAergic motor neurons promotes exit from dorsal bending}

We have shown that iMNs inhibit both ventral and dorsal muscles (Fig. 3). iMNs make inhibitory NMJs to ventral muscles, but they do not make synapses to dorsal muscles, or to eMNs that activate dorsal muscles (Fig. 1B; Fig. S1). This raises the possibility of extrasynaptic signaling.

Synaptic and extrasynaptic GABA inhibition involves ionotropic $\mathrm{GABA}_{\mathrm{A}}$ and metabotropic $\mathrm{GABA}_{\mathrm{B}}$ receptors, respectively ${ }^{24-26}$. Consistent with previous reports ${ }^{27,28}$, in newborn L1 larvae, the $\mathrm{GABA}_{\mathrm{A}}$ receptor (UNC-49) is present in ventral muscles and not in dorsal muscles (Fig. 5D). Along the ventral body, endogenously tagged UNC-49::GFP formed six clusters (Fig. 5D), reminiscent of the NMJ clusters by individual DD motor neurons (Fig. 3A. In the absence of $\mathrm{GABA}_{\mathrm{A}}$ (unc-49 mutant larva), inhibition of ventral muscles induced by iMN activation was significantly attenuated, but inhibition on dorsal muscles was only modestly reduced (Fig. 5A, B). Thus the $\mathrm{GABA}_{\mathrm{A}}$ receptor is critical for inhibition to ventral muscles, and less so for dorsal muscles.

In the absence of GABA $(g b b-2$ mutant larva), inhibition of dorsal muscles upon iMN activation was potently attenuated, whereas inhibition of ventral muscles was unaffected (Fig. 5A, B). Thus, $G_{A B A}$ receptors play a predominant role in dorsal relaxation. We found that the $\mathrm{GABA}_{\mathrm{B}}$ receptor, a GBB-1/GBB-2 complex, is more broadly expressed than the $\mathrm{GABA}_{\mathrm{A}}$ receptors. GBB-2::GFP is present in many neurons including eMNs, as well as in muscles (Fig. 5D).

Inhibition of dorsal muscles was not fully abolished in either 
A

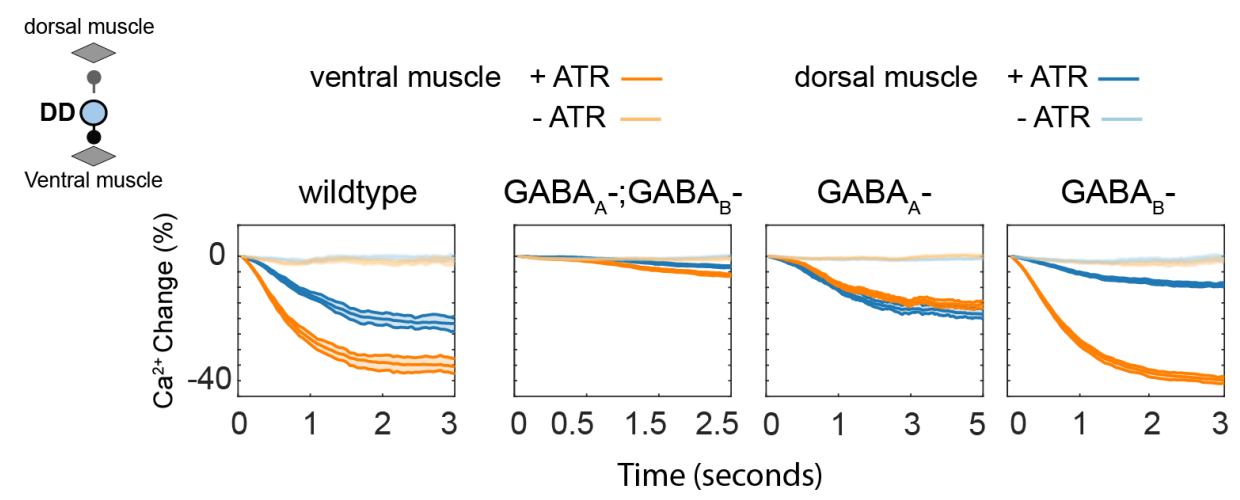

B

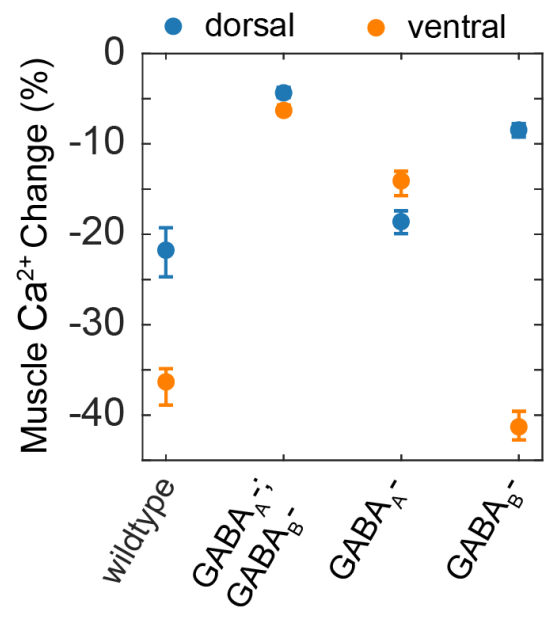

C

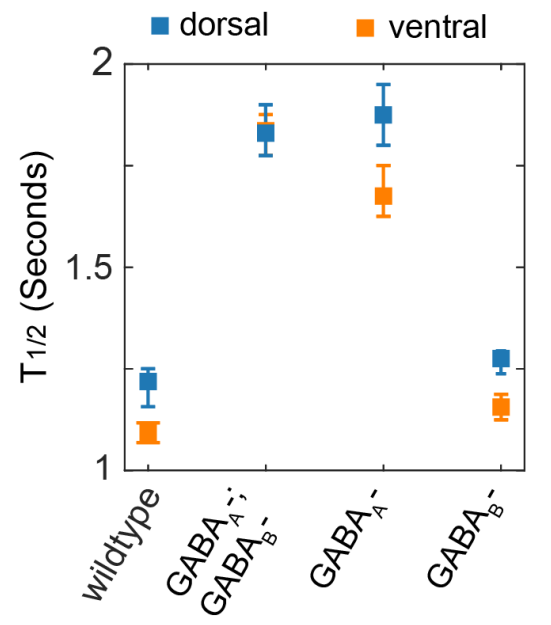

D

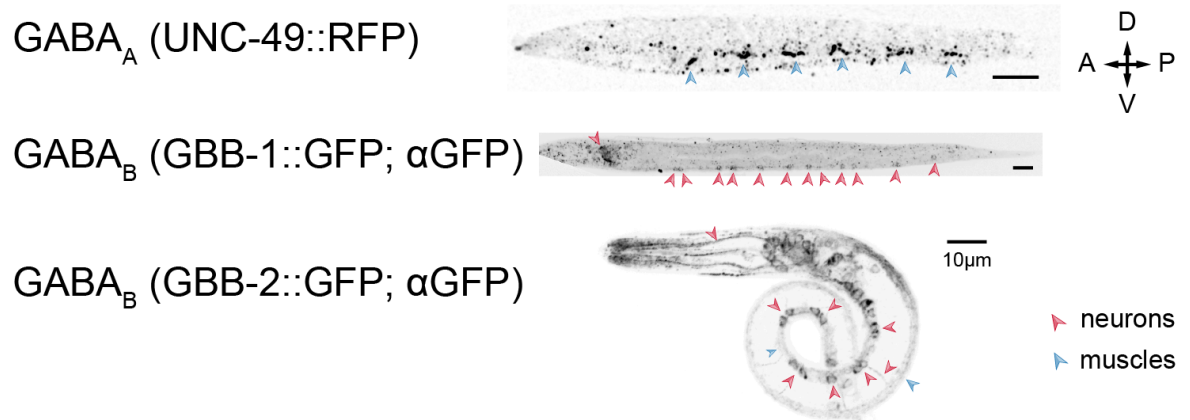

Figure 5. lonotropic and metabotropic GABA signaling inhibits ventral and dorsal muscles, respectively A. (inset) Schematic depicting simultaneous optogenetic activation of all iMNs and muscle calcium imaging. Y-axis plots percentage changes of the ventral and dorsal muscle activity from $t=0$. iMN activation reduced both dorsal and ventral muscle activities in wildtype $L 1$ larvae. The reduction was differentially attenuated in ionotropic $G_{A B A}$ and metabotropic $G A B A_{B}$ mutant larvae. For $G A B A_{A}$ mutants ( unc-49(e407): Control group (-ATR): 37 stimulation epochs from 22 larvae; Experimental group (+ATR): 51 stimulation epochs from 20 larvae. For GABA $A_{B}$ mutants (gbb-2(tm1165)): Control group (-ATR): 18 stimulation epochs from 6 larvae; Experimental group (+ATR): 39 stimulation epochs from 7 larvae. For double mutants: Control group (-ATR): 46 stimulation epochs from 12 larvae; Experimental group (+ATR): 76 stimulation epochs from 19 larvae. Lines and shades denote median and $95 \%$ confidence intervals, respectively. B Maximal activity changes in dorsal and ventral muscles in respective genotypes shown in $\mathbf{A}$. $\mathbf{C}$ Kinetics measured through the half-time of dorsal and ventral muscle activity changes in respective genotypes shown in A. D Expression patterns of endogenously tagged $G_{A B A}$ ::RFP receptors and GFP tagged fosmid reporters for two subunits of a heterologous $\mathrm{GABA}_{B}$ receptor complex, GBB-1::GFP and GBB-2::GFP.

$u n c-49$ or $g b b-2$ mutant larvae. Only in L1 larvae that lack both $\mathrm{GABA}_{\mathrm{A}}$ and $\mathrm{GABA}_{\mathrm{B}}$ receptors, $\mathrm{iMN}$ activation failed to inhibit dorsal and ventral muscles (Fig. 5A, B). Mutant L1 larvae that do not synthesize GABA unc-25) recapitulated this response (Fig. S5).

Despite making a minor contribution to dorsal muscle inactivation, $\mathrm{GABA}_{\mathrm{A}}$ receptors controlled the rate of inhibition in both ventral and dorsal muscles (Fig. 5C). Together, these results demonstrate that iMNs indirectly relax dorsal muscles. Extrasynaptic inhibition to eMNs works in concert with synaptic inhibition to ventral muscles to promote an exit after dorsal bending.

Therefore, in L1 larvae, motor neurons form a circuit where synaptic wiring drives dorsal bending. This circuit regulates its own exit from dorsal bending through a negative feedback in part by extrasynaptic GABA signaling. 
A

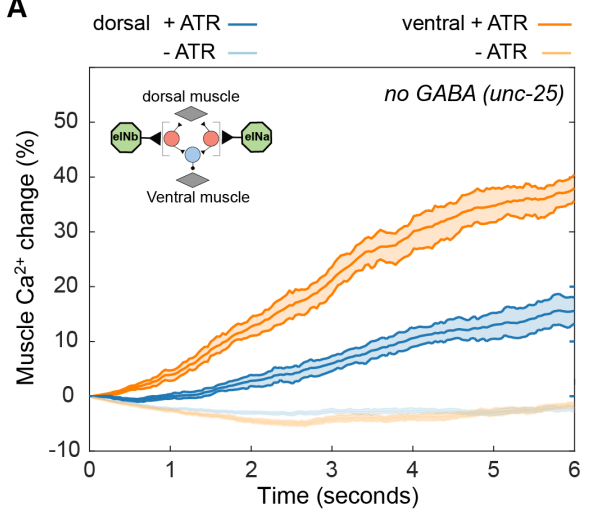

B

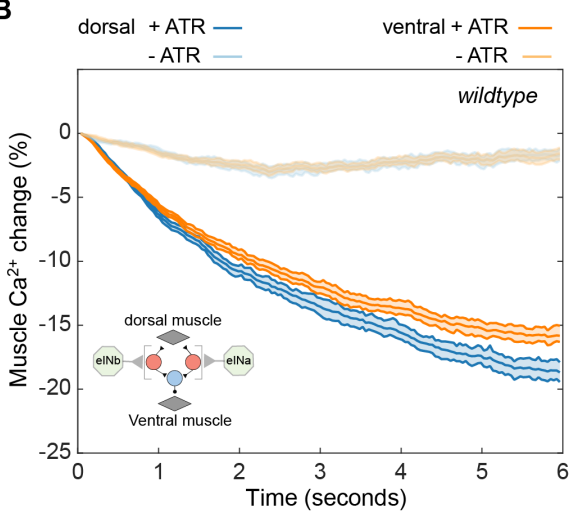

Figure 6. Cholinergic elNs underlie ventral muscle contraction A (inset) Schematic of simultaneous elN optogenetic activation and muscle calcium imaging in unc-25 L1 larvae. Y-axis plots percentage changes of the muscle activity from $t=0$. Activation of elNs by Chrimson elevated both dorsal and ventral muscle activity. Control group(-ATR), 51 stimulation epochs from 17 larvae; Experimental group(+ATR): 43 stimulation epochs from 12 larvae. B (inset) Schematic of simultaneous inactivation of all elNs and muscle calcium imaging in wildtype L1 larvae. Inhibition of elNs by GtACR2 reduced both dorsal and ventral muscle activity. Y-axis plots percentage changes of the muscle activity from $t=0$. Control group(-ATR): 28 stimulation epochs from 6 larvae; Experimental group(+ATR): 49 stimulation epochs from 13 larvae.

\section{Ventral muscle excitation requires cholinergic premotor interneurons}

A motor circuit to drive dorsal bending and its own exit does not produce ventral bending. Ventral bending requires ventral muscle contraction that occurs in synchrony with dorsal muscle relaxation. Other mechanisms must excite ventral muscles in order for the L1 motor circuit to transit from dorsal to ventral bending. To reveal this mechanism, we first considered the simplest possibility: a higher myogenic activity of ventral muscles compensates for the absence of motor neuron excitation. However, we found that optogenetic inhibition of the L1 larva's entire nervous system led to similarly silenced dorsal and ventral muscles (Fig. S6A). This shows that ventral muscle activity is not myogenic, but is driven by neurons.

To identify neurons that excite ventral muscles, we turned to optogenetic stimulation. Because ventral muscles receive inhibitory NMJs from iMNs, in wildtype larvae, pan-neuronal excitation activated dorsal muscles but inhibited ventral muscles (Fig. S6C, left panel). In L1 larvae that do not synthesize GABA (GAD/unc-25 mutant), however, we observed activation of both ventral and dorsal muscles (Fig. S6C, right panel). This assay was then applied to identify the neuron source for ventral muscle activation.

We next examined whether the excitatory input to ventral muscles is cholinergic. We found that optogenetic manipulation of all cholinergic neurons recapitulated the effects of pan-neuronal manipulation. Pan-cholinergic inhibition abolished activity in both dorsal and ventral muscles (Fig. S6B). Pan-cholinergic stimulation led to dorsal muscle excitation and ventral muscle inhibition in wildtype larvae (Fig. S6D, left panel), but activation of both dorsal and ventral muscles in (unc-25) mutant larvae (Fig. S6D, right panel). Thus, cholinergic neurons are responsible for ventral muscle activation.

We have shown that cholinergic eMNs do not innervate ventral muscles (Fig. 2). Axons of several premotor interneurons (eINs) are cholinergic ${ }^{29}$, and span the entire ventral nerve cord, making them the candidate source of ventral muscle excitation. Along the nerve cord, two eINs, AVA and PVC, make numerous synapses to all eMNs. Others (AVB, AVE, AVD) make synapses to AVA and PVC, and to one another (Fig. 1B).

We found that optogenetic inhibition of eINs by GtACR2 silenced both dorsal muscles and ventral muscles (Fig. 6B; Supplemental movie 9). In mutant larvae without GABA, optogenetic stimulation of eINs activated both dorsal and ventral muscles, with a stronger activation of ventral muscles (Fig. 6A; Supplemental movie 10). Patterns of dorsal and ventral muscle activity also differed: dorsal activity rise was in correlation with local curvature, whereas ventral activity rise was uniform along the body (Supplemental movie 10). Similar distinction was observed during optogenetic silencing of iMNs in wildtype larvae (Fig. 3D). These results suggest that ventral muscles may be uniformly activated by extrasynaptic cholinergic signaling from eINs.

To further determine whether eINs are required for ventral muscle activation, we ablated them using miniSOG. All eIN-ablated L1 larvae exhibited diminished ventral muscle activity, but with residual dorsal muscle activity (Fig. 7A; Supplemental movie 11). When inhibitory input to ventral muscles was removed in unc-25 mutant larvae, ablation of eINs also reduced ventral muscle but not dorsal muscle activity (Fig. 7B; Supplemental movie 13). Reduced ventral muscle activity confirms that eINs are required for ventral muscle excitation. Persistent dorsal muscle activity is consistent with the presence of endogenous eMN activity, as previously observed in the adult circuit ${ }^{30,31}$. These results establish the requirement of eINs in stimulating ventral muscles.

\section{Cholinergic premotor interneurons activate ventral muscles via extrasynaptic signaling}

Because eINs do not make synapses to ventral muscles (Fig. 1B; Fig. S1), they may activate ventral muscles by ephaptic coupling, or by extrasynaptic mechanisms that require neurotransmitter release.

We distinguished these two possibilities by assessing the effect of blocking synaptic vesicle fusion using tetanus toxin $(\mathrm{TeTx})^{32}$. When vesicular release from all eINs was blocked, activity in both dorsal and ventral muscles was reduced (Fig. 7C; Supplemental movie 14). Vesicular release is needed for eINs to activate dorsal muscles because they innervate eMNs, as expected. Reduced ventral muscle activation, 
however, demonstrates that neurotransmitter release is also needed for eINs to activate ventral muscles. Similar to effects of eIN ablation, ventral muscle activity was more severely reduced than dorsal muscles (Fig. $7 \mathrm{C}$, middle panel). These results argue for the requirement of vesicular release of acetylcholine to drive ventral muscle activation. Extrasynaptic accumulation of acetylcholine is also consistent with the uniform increase of ventral muscle activity during eIN stimulation.

These results suggest that cholinergic eINs stimulate ventral muscles through extrasynaptic acetylcholine accumulation.

\section{L1 motor circuit orchestrates dorsal-ventral symmetry by anti-phasic entrainment}

Our data demonstrated that synaptic wiring among motor neurons is built to make only dorsal bends. But with ventral muscles tonically activated by eINs, the same wiring can entrain ventral muscles to generate complementary ventral bends. An implication of this model is that ventral muscle excitation takes places independently of motor neurons, but synaptic wiring among motor neurons is required to generate coordinated dorsal-ventral bending.

Consistent with this implication, blocking synaptic transmission from eMNs and eINs by TeTx led to opposite effects on dorsal and ventral muscles (Fig. 7). Eliminating eINs or their synaptic output resulted in dorsal muscle activity being higher than ventral muscle activity (Fig. 7A-C). Eliminating eMN output led to ventral muscle activity being higher than dorsal muscle activity (Fig. 7D).

Behaviorally, however, only dorsal muscle's calcium signals predict a crawling larva's bending pattern, reflecting direct input from eMNs. When eINs or their synaptic output were eliminated, regardless of the absolute level of residual dorsal activity, they led to a persistent bias for dorsal bending across body segments, in wildtype as well as unc-25 mutant larvae (Fig. 7A-C; Supplemental movie 11-13).

When eMN synaptic output was blocked, regardless of a high ventral muscle activity (Fig. 7D), L1 larvae exhibited no consistent dorsal or ventral bending bias across body segments (Fig. 7D; Supplemental movie 14). This coincides with a spatially uniform calcium signal in their ventral muscles that lacked correlation with curvature (Fig. 7D). The lack of correlation is similar to the calcium pattern of ventral muscles in the unc-25 mutant larvae (Fig. 3D).

\section{A computational model: generating a symmetric motor pattern with an asymmetric circuit}

How bending is generated in each segment is illustrated in Fig. 8A. In L1 larvae, dorsal and ventral muscles have similar excitability (Fig. S6). Rhythmic dorsal bending is directly mediated by oscillatory activity of cholinergic motor neurons. Cholinergic motor neurons simultaneously activate GABAergic neurons that relax ventral muscles. The net effect is a dorsal bend (Fig. 8A, i).

Rhythmic ventral bending is not mediated by its own oscillator, but by anti-phasic entrainment to the oscillator that drives dorsal bends. Ventral muscles are uniformly excited by extrasynaptic acetylcholine from premotor interneurons. This permits ventral bends to occur when and where the dorsal oscillator's activity is low (Fig. 8A, iii).
Transitions between dorsal bending and ventral bending are also facilitated by negative feedback within each segment: GABA released by inhibitory motor neurons relaxes ventral muscles and extrasynaptically inhibits excitatory motor neurons that drive dorsal bending (Fig. 8A, ii). Thus, in L1 larvae, the same set of motor neurons drive both dorsal and ventral bends. This is in contrast to the adult, which has two symmetrically wired motor subcircuits that separately drive dorsal and ventral bends.

We developed a phenomenological model that describes L1 motor circuit dynamics (Fig. 8B) (see Methods). Similar to previous work in the adult motor circuit ${ }^{31}$, the $\mathrm{eMN}$ is modeled as an oscillator $\left(V_{d i}\right)$ with calcium and potassium conductances. It provides direct input to dorsal muscles $\left(M_{d i}\right)$ through an excitatory synapse. The eMN relays a copy of this oscillation to ventral muscles $\left(M_{v i}\right)$, inverting its sign through an inhibitory synapse $(D D)$. When dorsal and ventral muscles have the same resting membrane potential, the inhibitory synapse rectifies dorsal oscillations. This produces alternating dorsal-ventral bends with a deeper dorsal bend (Fig. 8C, lower panel). An extrasynaptic excitatory input to ventral muscles $(e I N)$ alleviates the dorsal bias (Fig. 8C, upper panel).

We modeled propagating bending waves through proprioceptive input between adjacent eMNs $\left(\kappa_{i}\right)$. As in previous models ${ }^{31,33}$, directional wave propagation is encoded by proprioceptive input where the eMN in segment $i$ receives curvature-dependent input from $i-1$ (for forward locomotion) or from $i+1$ (for backward locomotion). Net bending is calculated from the extent of dorsal and ventral muscle activation in each segment (see Methods). We obtained parameters by fitting the model outputs to key experimental findings on dorsal and ventral output symmetry (Fig. 7).

Without the inhibitory synapse to ventral muscles, this model predicts stalled wave propagation on the ventral side. This fully recapitulates the lack of correlation of ventral muscle activity with bending of GABA-(unc-25) mutant larvae (Fig. 3F). Perturbation of the inhibitory synapse in this model generates bending with a ventral bias across the body. Behaviorally, while $u n c-25$ mutants exhibit a ventral bias, they do also generate dorsal bends (Supplementary movie 15). Our model can recapitulate this phenomenon by adjusting the extrasynaptic input to ventral muscles. This suggests that premotor interneurons may receive a feedback from the downstream motor circuit.

Elimination of extrasynaptic input in this model generates dorsal bending across the body, which recapitulates the behavior of eIN-ablated larvae (Fig. 7A-C). Compared to a simpler model, where ventral muscles are spontaneously active, an extrasynaptic input to ventral muscles confers more flexibility for bending waves. This functional configuration offers a temporary solution for symmetric bending before the motor circuit matures. 
bioRxiv preprint doi: https://doi.org/10.1101/2021.09.21.461278; this version posted December 21, 2021. The copyright holder for this preprint (which was not certified by peer review) is the author/funder, who has granted bioRxiv a license to display the preprint in perpetuity. It is made available under aCC-BY-NC-ND 4.0 International license.

A
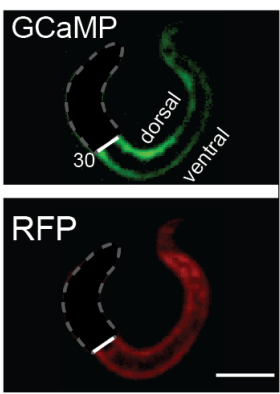

head $=1$; tail $=100$

B
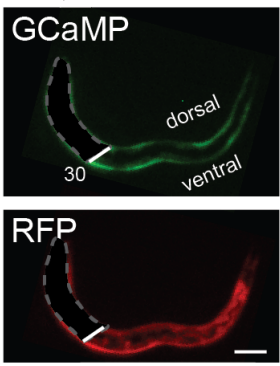

head $=1$; tail $=100$

C
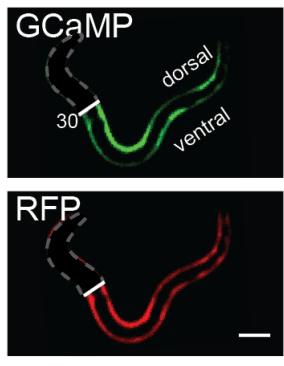

head $=1 ;$ tail $=100$

D
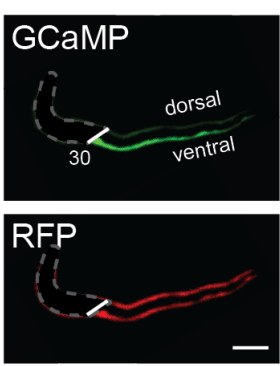

head $=1 ;$ tail $=100$
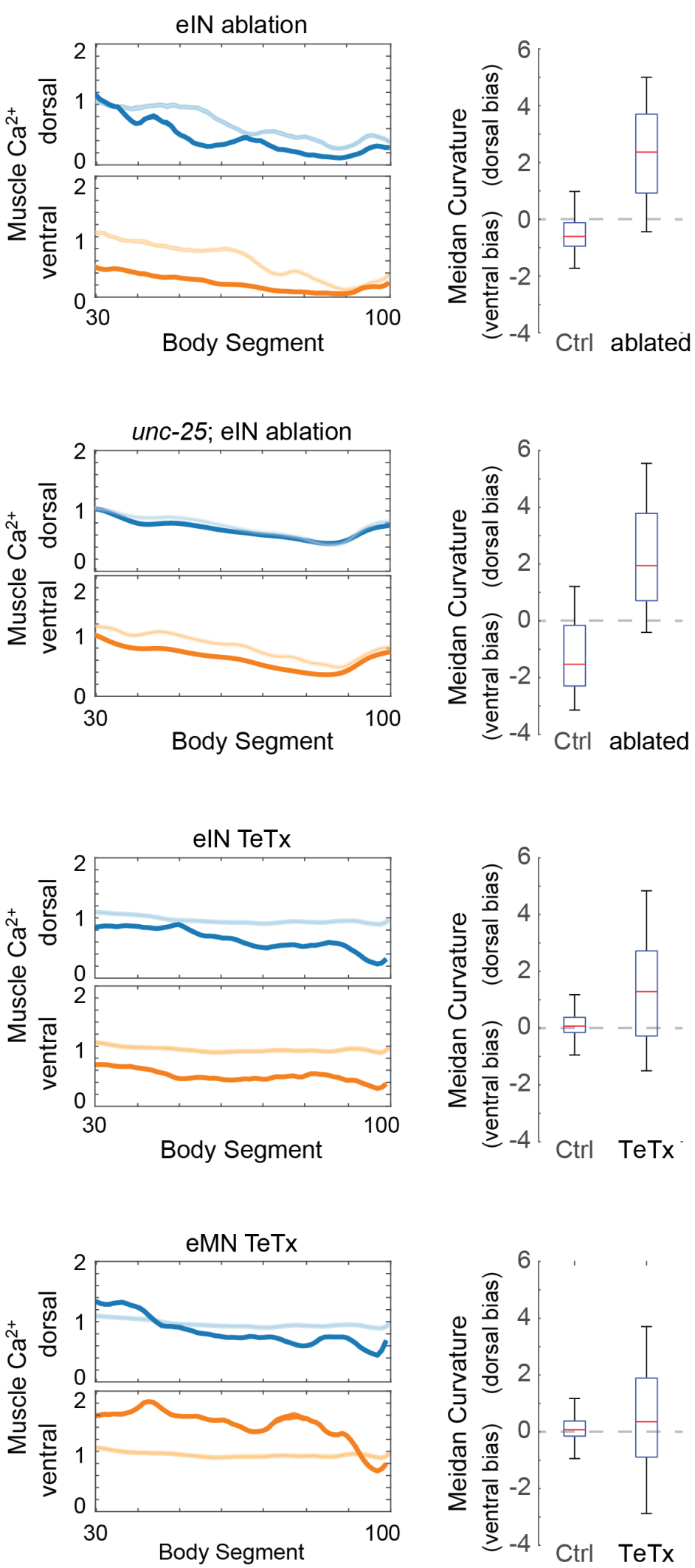

Figure 7. Extrasynaptic transmission from elNs is required for ventral bending A. Muscle activity and body curvature of L1 larvae without elNs. (left panel) Example images of muscle GCaMP::RFP; (middle panel) Average dorsal (top) and ventral (bottom) muscle activity. Lighter colors represent activities of the mock-ablated group; (right panel) Median curvature across the body (segments 33-95) of elN ablated group compared with the control group (ctrl). eIN ablation led to stronger reduction of ventral activity; these larvae exhibited a bias for dorsal bending along the body. B. Same as A, but for mutant L1 larvae without GABA GAD/unc-25(e156). Upon elN ablation, L1 larvae exhibited reduced ventral muscle activity and a bias for dorsal bending across the body. C. Muscle activity and body curvature of L1 larvae where synaptic vesicle release from elNs was constitutively blocked by TeTx. (left panel) Example images of muscle GCaMP::RFP; (middle panel) Average dorsal (top) and ventral (bottom) muscle activity. Lighter colors show activities of L1 larvae without TeTx (control group); (right panel) Median curvature across the body (segments 33-95) of synaptic vesicle release blocked group compared with the control group (ctrl). Blocking vesicle release from elNs also resulted in a reduction in ventral muscle activity and a bias for dorsal bending across the body. $\mathbf{D}$. Same as $\mathbf{C}$, but with L1 larvae expressing TeTx in eMNs. Blocking synaptic vesicle release from eMNs resulted in a higher ventral muscle activity, but not a consistent bias towards either ventral or dorsal bending across the body. 
bioRxiv preprint doi: https://doi.org/10.1101/2021.09.21.461278; this version posted December 21, 2021. The copyright holder for this preprint (which was not certified by peer review) is the author/funder, who has granted bioRxiv a license to display the preprint in perpetuity. It is made available under aCC-BY-NC-ND 4.0 International license.

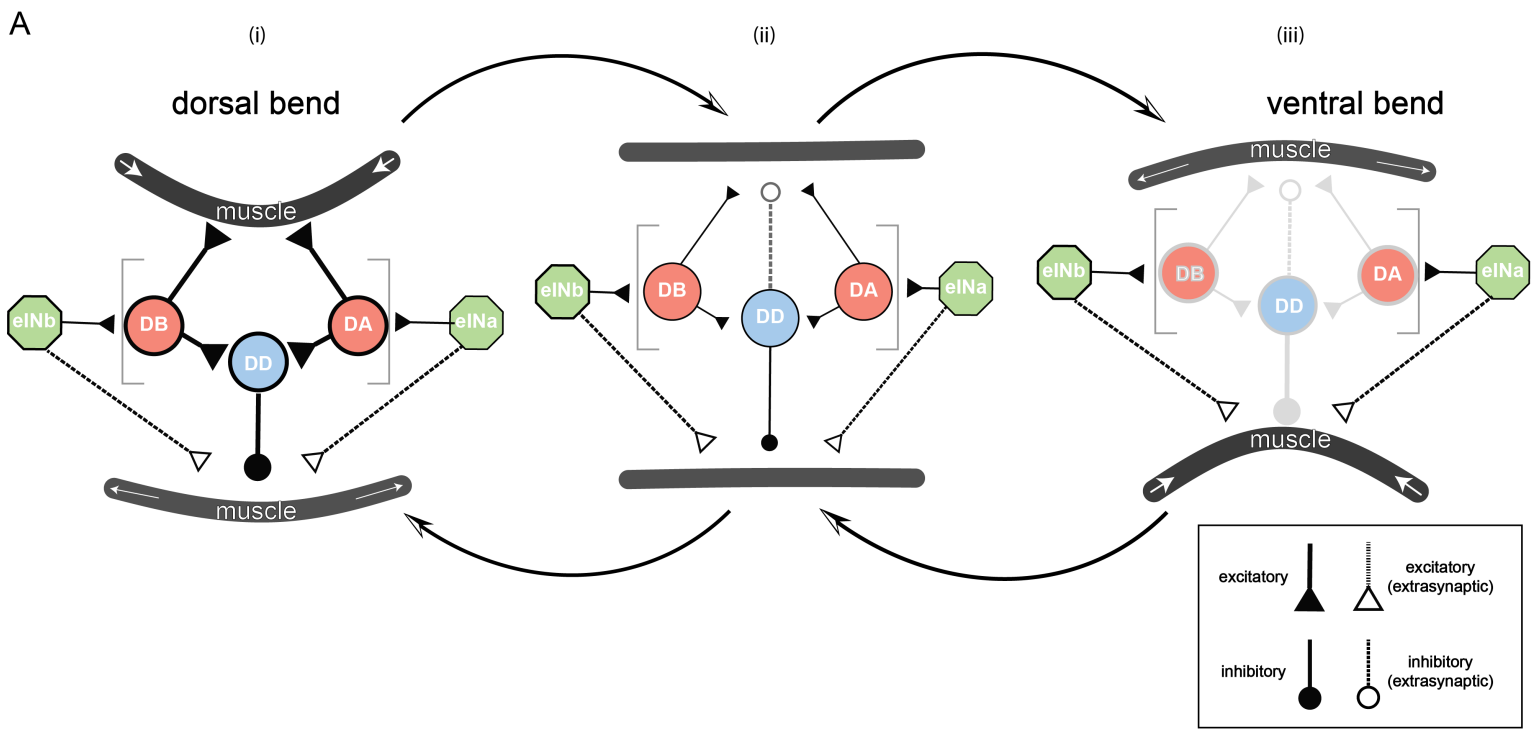

B

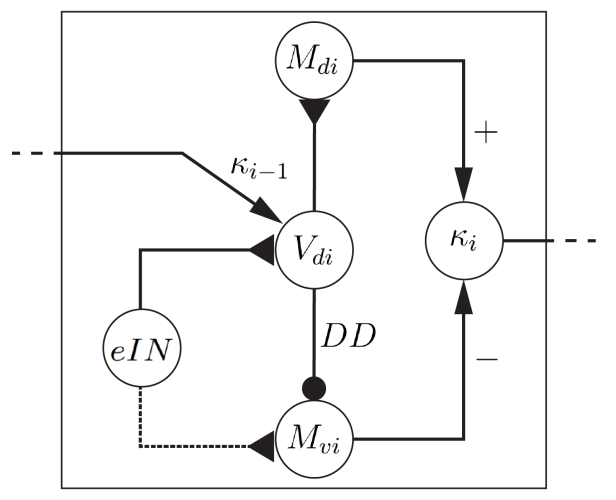

C
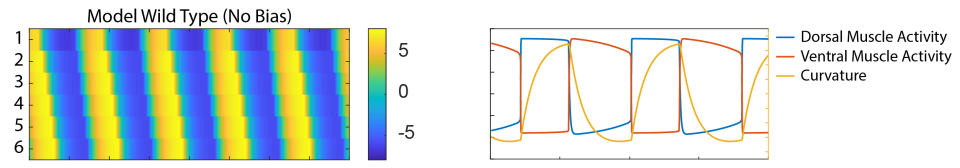

Model DD Ablation (Ventral Bias)

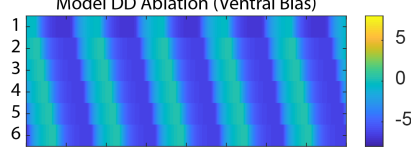

Model Extrasynaptic Input Ablation (Dorsal Bias)
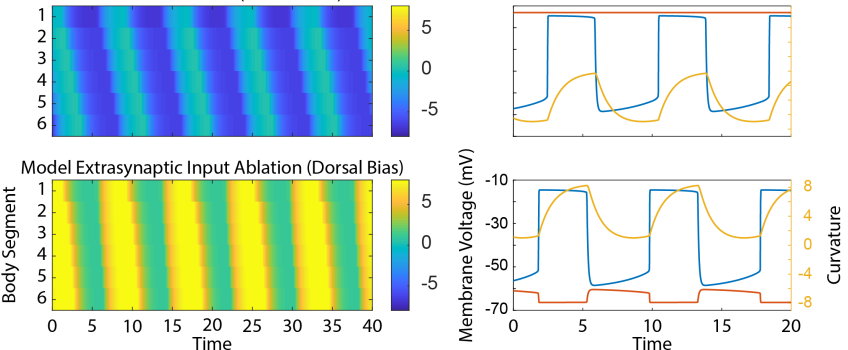

Figure 8. A computational model of the L1 motor circuit A L1 larvae integrate synaptic transmission and extrasynaptic transmission to generate alternating body bends. Schematic depicts transitions between three bending phases. (i) Synaptic wiring from the eMN and iMN contracts dorsal muscle and relaxes ventral muscle, generating a dorsal bend. (ii) Reduced synaptic transmission from the eMN and inhibitory feedback from the iMN reduces dorsal contraction and ventral relaxation, promoting exit from a dorsal bend. (iii) Further reduction of eMN activity removes inhibition on ventral muscle, allowing extrasynaptic excitation from the elN to generate a ventral bend. B Schematic of the computational model at one body segment. $V_{d i}, M_{d i}, M_{v i}$ denote membrane potentials of the eMN, dorsal muscle and ventral muscle at segment $i$, respectively. $\kappa_{i}$ is the curvature at segment $i . \kappa_{i-1}$ represents the proprioceptive input from the anterior segment. $D D$ denotes the implicit inhibitory synapse on ventral muscle. Inverted and circular arrowheads denote excitatory and inhibitory synapses, respectively. Dotted arrow denotes extrasynaptic excitation. Regular arrowheads represent mathematical operations. C (left) Curvature kymographs from simulations in different conditions. (right) Time-series of dorsal-ventral muscle activity and curvature at mid-body (segment 3). (top) Simulation of the full circuit produces traveling waves along the body. (middle) Simulation of the circuit without inhibitory synapse produces traveling waves only in dorsal muscles. (bottom) Simulation of the circuit without extrasynaptic input from the eIN leads to severe reduction of ventral muscle activity and strong bias for dorsal bending. 


\section{Discussion}

It is often assumed that symmetric motor circuits are necessary for symmetric motor outputs. C. elegans is born only with motor neurons that are wired analogously to the motor subcircuit for dorsal bending in adults. Over the course of larval development, extensive neurogenesis and rewiring adds the other motor subcircuit for ventral bending and eliminates asymmetries in the layout of the newborn circuit.

The larva and adult generate the same gait despite profound anatomical differences. We show that L1 larvae achieve a symmetric gait by using the same motor circuit to drive dorsal bending and to antiphasically entrain ventral bending. $C$. elegans has a strong drive for a stable motor output during development. .

Circuit degeneracy maintains an animal's motor patterns

The $C$. elegans motor circuit presents a profound example of circuit degeneracy: a functional output has multiple network solutions ${ }^{14}$. Previous examples include similar motor patterns exhibited by two molluscs with substantially different functional connectivity ${ }^{34}$, and maintenance of a pyloric circuit's output at different temperatures by varying parameters of synaptic connections ${ }^{35}$. Circuit degeneracy has been proposed to play a critical role in population diversity, adaptability to changing environments, and specie evolution.

Our results demonstrate that degeneracy also minimizes functional disruption to locomotion during development, representing an intrinsic drive to maintain stable motor output in response to ecological demand.

Extrasynaptic signaling enables an asymmetric L1 motor circuit to generate symmetric motor output

L1 larva's alternative solution for symmetric bending requires extrasynaptic signaling at multiple layers of its motor circuit.

Dorsal bending is driven by a configuration that resembles the original half-center model proposed for flexor-extensor coordination during limb movement ${ }^{1}$ : synaptic wiring of excitatory and inhibitory motor neurons coordinates dorsal muscle excitation with ventral muscle relaxation.

In contrast, ventral bending is generated by anti-phasic entrainment to the rhythmic activity of this dorsal 'half-center'. For this to occur, it is necessary for ventral muscles to be uniformly activated. Acetylcholine from premotor interneurons, which accumulates and acts extrasynaptically, fulfills this role.

Intrinsic oscillatory activities of excitatory motor neurons underlie transitions from dorsal to ventral bending, but GABA release from inhibitory motor neurons facilitates and modulates this transition. Acting extrasynaptically, this negative feedback inhibits excitatory motor neurons and promotes dorsal muscles to relax after contraction.

Thus extrasynaptic signaling is necessary for the asymmetric motor circuit to create symmetric output.

\section{Extrasynaptic signaling as an adaptive strategy for a developing motor circuit to create mature behavior}

Extrasynaptic accumulation of fast neurotransmitters may result from vesicular release at extrasynaptic sites, or by extracellular diffusion of released neurotransmitters ${ }^{36}$. We favor the diffusion mechanism for extrasynaptic activation of ventral muscles, because we did not observe an overt non-synaptic vesicle accumulation in the EM volume of newborn L1 larvae.

Extrasynaptic signaling is widely observed in other systems ${ }^{37-40}$. It is one mechanism for neuromodulation, which allows hard-wired mature circuits to modify and adapt their output to different contexts ${ }^{41,42}$. Our results reveal that during early development, extrasynaptic transmission is essential, functionally compensating for the absence of an entire motor subcircuit. As larvae mature, this role is taken over by postembryonic motor neurons and synaptic wiring.

Not all extrasynaptic transmission is replaced as the motor circuit matures. Extrasynaptic feedback from GABAergic motor neurons to excitatory motor neurons persists in the adult $^{24-26}$. It may play similar roles in both dorsal and ventral motor subcircuits.

Extrasynaptic mechanisms may work particularly well in the larva because of its small size. In a short-lived animal, acquiring full mobility at birth is of probable fitness benefit. Extrasynaptic signaling thus represents an adaptive strategy for development.

\section{Neurons adopt new functions to compensate for structural changes}

In small circuits, individual neurons often adopt multiple functions to expand their behavioral repertoires ${ }^{43,44}$. Multi-functionality is generally considered to be a property of mature neural circuits.

In the adult, cholinergic motor neurons are rhythm generators for forward and backward movement ${ }^{30,31,45}$. Cholinergic premotor interneurons signal to switch the these two modes of locomotion ${ }^{12,31}$. Our calcium imaging of premotor interneurons and behavioral effects upon their activation (Fig. S7) showed that this role is likely conserved across development. However, premotor interneurons assume an additional role in the L1 larva: they directly activate ventral muscles. This excitation is independent of motor neurons. Neither is this excitation the consequence of positive feedback from cholinergic motor neurons, because cholinergic motor neurons specifically activated dorsal muscles.

The essential role of premotor interneurons in ventral muscle excitation is likely temporary: the expression pattern of acetylcholinesterase encoding genes, whose enzymatic activity determines extrasynaptic acetylcholine buildup, exhibits dorsal-ventral asymmetry in the newborn larva. Asymmetry disappears in adults, coinciding with the emergence of ventral muscle-innervating motor neurons of the adult circuit ${ }^{46}$.

Thus, a neuron's flexibility to adopt multiple roles also provides a means to compensate for a circuit's developmental immaturity.

\section{An adequate but temporary solution for a developing motor circuit}

How L1 larvae generate a symmetric gait with an immature, asymmetric circuit has been an enigma. Several hypotheses have been proposed ${ }^{47,48}$, but our experimental findings are inconsistent with these models.

Prior models assumed ventral bending to be myogenic. Our model incorporates a neural source of ventral muscle excitation established by experimental findings, providing a more flexible solution. Because the relative strength of synaptic and 
extrasynaptic inputs from premotor interneurons - the source of ventral muscle excitation - can be adjusted separately, this model confers flexibility and a range of possible gait patterns with balanced or imbalanced dorsal-ventral bends.

In this L1 model, dorsal and ventral muscles are driven by the same oscillators. Consequently, their activation is not fully independent of each other. This implies that synaptic input to dorsal muscles alone determines wave propagation. This recapitulates the experimental outcome of decoupling of dorsal and ventral muscle activity: when we removed synaptic output of dorsal oscillators, or the inhibitory synapse that relays this oscillation to ventral muscles, ventral calcium signals did not propagate and did not correlate with bending.

This configuration differs from the adult, where both dorsal and ventral muscles are driven by dedicated motor subcircuits. Differential activation of dorsal and ventral muscles might have advantages in the worm's natural habitat, where it would enable more robust exploration in a $3 \mathrm{D}$ environment ${ }^{49}$. Thus, L1 larva's configuration offers an adequate but temporary solution.

\section{Methods}

\section{Constructs, transgenes and strains}

A list of constructs, transgenic arrays, and transgenic strains acquired and generated for this study is summarized in the Supplemental text.

\section{Electron microscopy of a mid-L1 stage larva}

Serial electron microscopy was performed as previously described $^{50,51}$. The $\mathrm{L} 1$ larva reconstructed here was at mid-L1 stage (approximately 5-6hr) prior to the beginning of post-embryonic neurogenesis. It covers over half of the body. It was manually serial sectioned at $50 \mathrm{~nm}$ thickness and imaged by TEM at $0.7 \mathrm{~nm} /$ pixel. Images were stitched into $3 \mathrm{D}$ volumes using TrakEM2 Fiji plugin ${ }^{52,53}$, and annotated after skeleton tracing in the CATMAID ${ }^{54}$. A full reconstruction of the ventral and dorsal nerve cords of an newborn L1 larva (1hr) revealed the same pattern and is described as part of another study (Mucalhy et al. in preparation).

\section{Synchronization of young L1 larvae}

L1 larvae used for most behavioral and imaging experiments were collected as follows. On the day of experiment, up to fifty 3-fold stage eggs from plates with well-fed gravid adults were transferred to a new plate seeded with OP50. Eggs were allowed to hatch for $1 \mathrm{hr}$ at $22.5^{\circ} \mathrm{C}$ and unhatched eggs were removed. All experiments were carried out within 5-6 hours afterwards to ensure that they had not integrated postembryonically derived motor neurons. ${ }^{15}$.

\section{Crawling assays and analyses}

Free crawling on culturing plates For most recordings (Fig. 7), individual L1 larvae hatched on a thin lawn of OP50 on a NGM plate were recorded directly from the plate using a dissecting microscope (Zeiss V16). Some recording were performed using a customized wide-field compound microscope ${ }^{12}$ with larva movements manually tracked during recording. For some experiments, larvae were maintained until adulthood to verify successful ablation of neurons (Fig. 7A-D).

Imaging analyses were carried out using in-house MATLAB scripts to analyze both body curvature and velocity. Orientation (head, tail, ventral, and dorsal) were manually assigned in the first frame of the recording and tracked automatically afterwards. Contours of L1 larvae were segmented and divided into 100 segments along the anterior-posterior axis, and errors fixed by manual curation.

On plate optogenetic stimulation For each set of optogenetic experiments, the control and experimental group contained animals of the same genotype, with the control group cultured on plates without all-trans retinal (ATR $)^{55}$. All larvae were recorded on unseeded, no ATR plates.

Animals in the experimental group was cultured on NGM plates supplied with $0.1 \mathrm{mg} / \mathrm{ml}$ ATR for at least two generations. Individual $\mathrm{Ll}$ larvae were transferred to and recorded using the same unseeded imaging plate as described above, typically for $75 \mathrm{sec}$ at $26 \mathrm{~Hz}$. L1 larvae from the control group was recorded on another unseeded imaging plate.

All animals were subjected to stimulation from an LED light source controlled by a customized ImageJ plugin ${ }^{12}$. The stimulation protocol was 10 second light-off (pre-stimulation), 4 consecutive epochs of stimulation consisting of 10 second light-on and 5 second light-off, followed by 10 second light-off (post-stimulation). Image analyses were carried out using customized MATLAB scripts.

\section{Swimming assays}

Recording of swimming wildtype L1 larvae was performed in a PDMS miniature well (fabricated Hang Lu lab, Georgia Institute of Technology, USA) or a simple chamber made by a thin circle of vaseline between two glass slides. A droplet of M9 was mounted onto the center of the well or chamber, and L1 larvae were transferred into the M9 droplet. With a cover-slip mounted, the PDMS well was placed on a glass slide and recorded with a $4 \mathrm{x}$ objective with the compound microscope (above) for $68 \mathrm{sec}$ at $26 \mathrm{~Hz}$, or with a $10 \mathrm{X}$ objective with a dissecting microscope (Zeiss) for 5-10 minutes at $10 \mathrm{~Hz}$.

In Figure 1D, body postures were manually scored frame-by-frame. Ventral side of the larva was identified by the presence of anus and/or the gonad. A dorsal-biased bending posture was defined by a convex towards the dorsal side from the neck $(30 \%)$ to the tail. A ventral-biased bending posture was defined by a convex towards the ventral side from the neck $(30 \%)$ to the tail. Other postures include both dorsal and ventral bending and were deemed 'unbiased'. Frames where neither the anus nor the gonad could be confidently identified were discarded. The total number of frames each larva spent in one of three postures was normalized against the total number of frames for each recording.

\section{Photo-ablation by miniSOG}

Transgenic animals that express blue-light activated miniSOG protein were used in these experiments, performed and verified as described in $^{30}$.

Briefly, to visually examine ablation efficiency, miniSOG was co-expressed with SL2-RFP to tag the to-be-ablated neurons and neurites. Cells that activated apoptosis usually appear vacuolar after the light treatment. But the complete disappearance of soma and neurites could only be observed towards the end of the larval stage. 
A home-made chamber, designed and made by Taizo Kawano and Thomas Sun (Lunenfeld-Tanenbaum Research Institute, Canada), delivers the blue LED light, and dissipates heat and supplies oxygen by ventilation during illumination. For each experiment, $1 \mathrm{hr}$ post-hatching larvae on a seeded plate were placed in the chamber upright with its lid removed. The optimal exposure time was experimentally determined for each strain to ablate cells without arresting larva growth. The eIN ablation strain required 30 minute exposure. After illumination, animals were allowed to recover in darkness for 4 hours before behavioral and calcium imaging experiments.

After each larva was imaged, it was recovered and transferred onto individual plates to grow for two days. Only data recorded from those with absence of RFP in targeted neurons after two days were used for analyses.

\section{Calcium imaging of neurons}

L1 Larvae expressing GCaMP6s::RFP fusion in neurons were used in these experiments. Recordings were acquired on a customized LED-powered wide-field tracking compound microscope with L1 larvae slowly crawling on $2 \%$ agarose pads under coverslips. Dual-color recording of GCaMP6s and mCherry was performed using an in-house ImageJ plugin as described $^{12,56}$. L1 larvae were mounted with either a small droplet of M9 or halocarbon oil. Recordings were performed with $40 \mathrm{x}$ or $63 \mathrm{x}$ objectives, at $26 \mathrm{~Hz}, 20 \mathrm{~Hz}$ or $10 \mathrm{~Hz}$, for $68 \mathrm{~s}$, 90 s or 180 s, typically by manual stage tracking.

Calcium imaging analyses was carried out using a MATLAB script modified from ${ }^{57}$ that tracks individual regions of interest (ROI). Intensity and displacement of ROIs in the green and red channels were used to calculate calcium dynamics, velocity, and bending angles.

\section{Calcium imaging of muscles}

L1 Larvae expressing GCaMP3 and tagRFP separately in body wall muscles were used in these experiments. Some recordings (Fig. 1D) were acquired for L1 larvae slowing crawling on $2 \%$ agarose pads under coverslips, as for neuronal calcium imaging as above. Other recordings (Fig.7A-D) were performed with unconstrained L1 larvae crawling on 2\% agar plates made with S-basal buffer. Images were acquired with a Nikon spinning disk confocal microscope operating in a wide-field mode with a $10 x$ objective at 10fps. The setup was equipped with a dual view system and Andor Zyla VSC-07720 camera. Toptica's multilaser system was used for illumination at $10 \mathrm{~mW}$ power.

Post-imaging analyses used a customized MATLAB script. The head and tail are manually assigned in the first frame of the recording and tracked afterwards. The contour of the worm is divided into 100 segments along the anterior-posterior axis and further segment into the dorsal and ventral segments across the mid-line. Averaged intensity for pixels within each segment is used as proxy for the activity for this muscle segment. Bending curvatures were derived from angles between each segment.

To calculate the phase difference, we first used the Hilbert transform as implemented by MATLAB's hilbert function to obtain an analytical representation of the signal. The difference between the phase angles obtained from the analytical representation of muscle activities and curvatures was used to generate phase difference histograms.

\section{A simple strategy for simultaneous optogenetic stimulation and calcium imaging}

Simultaneous functional imaging and optogenetic stimulation is technically challenging due to the overlapping excitation spectra for opsins and genetic calcium sensors. Two strategies to address this issue are to spatially separate the illumination ${ }^{58,59}$ or to attempt spectral separation of opsins and calcium sensors ${ }^{59-64}$.

We approached simultaneous optogenetic stimulation and calcium imaging with a simpler goal. Because calcium readout exhibits significant latency compared to the light response of opsin channels, and opsins can be activated by the same illumination spectra for GCaMP and RFP, the initial change of calcium signals should already reflect the cell's response to opsin stimulation. We validated this strategy with a characterized connection (cholinergic motor neurons to their downstream GABAergic motor neurons in adults), and then applied it to examine the complete L1 motor circuit.

The following protocol conducts bi-directional (activation and inhibition) functional manipulation with any combination between all tested opsins (ChR2, Chrimson, Halorodopsin, GtACR2) and calcium sensors (GCaMP::RFP and chameleon). It can be carried out on wide-field fluorescent as well as confocal microscopes. The following protocol is not meant to probe dynamics of circuit connectivity. It is a simple but effective assay to establish causality and functional connectivity between neurons.

We used the same wide-field fluorescent microscope for calcium imaging. Strains that express opsins in upstream cells and calcium sensors in downstream cells were cultured on all-trans retinal (ATR)-supplemented plates for multiple generations. A control group was cultured on plates without ATR. For experimental groups, particularly strains generated from the Cre-loxP system, animals were cultured on plates supplied with higher concentration ATR $(250 \mu \mathrm{M})$ to increase the strength of optogenetic stimulation. On the day of experiments, L1 larvae were mounted on slides as described for neuron calcium imaging, except that they were immobilized by limited M9 solution. One minute after samples were mounted, recording was carried out initially without the LED light. During the recording, the LED light (serving both for calcium imaging and opsin stimulation) was turned on and off, with an on phase longer than 3 seconds, and off phase ranging from 5-20 seconds. Sampling rates ranged from $10-26 \mathrm{~Hz}$. Calcium imaging analyses were carried out as described above. Only the first 3-6 second recording for each epoch was used for analyses.

\section{A computational model}

We modeled each segment of the L1 motor circuit using the equations below (1-5), which is schematized in Fig. 8B. The model is inspired by the Morris-Lecar model of the Barnacle giant muscle fiber ${ }^{65}$. It's a reduced order nonlinear model with an instantaneous calcium current, a potassium current with an activation variable, and a passive leak current. A similar model was used previously to study adult $C$. elegans excitatory motor neurons ${ }^{31}$. We model 6 segments coupled to each other proprioceptively, corresponding to the 6 DD NMJs. $V_{d i}, M_{d i}, M_{v i}$ are the membrane potentials for the eMN, dorsal muscle and ventral muscle in segment $i$ respectively. $n_{i}$ is the 
activation variable for the slow potassium current and $\kappa_{i}$ is the segment's curvature. We implicitly model the activity of DD iMNs via an inhibitory synapse on ventral muscles by eMNs. We did not include the extrasynaptic inhibition to dorsal muscles in our model, because it was not necessary for the generation of a symmetric oscillatory gait. This also recapitulates our experimental findings, where inhibitory feedback on to dorsal muscles is modulatory and $g b b-1$ mutant larvae did not exhibit overt motor defects.

$$
\begin{aligned}
C_{m} \frac{d V_{d i}}{d t}=-g_{L}\left(V_{d i}-E_{L}\right)-g_{C a} m_{\infty}\left(V_{d i}\right)\left(V_{d i}-E_{C a}\right) \\
-g_{K} n_{d i}\left(V_{d i}-E_{K}\right)+c \kappa_{i-1}+I_{S Y N+}^{V_{e I N}} \\
\tau_{n} \frac{d n_{d i}}{d t}=-n_{d i}+n_{\infty}\left(V_{d i}\right) \\
\tau_{u} \frac{d M_{d i}}{d t}=-M_{d i}+I_{S Y N+}^{V_{d i}} \\
\tau_{u} \frac{d M_{v i}}{d t}=-M_{v i}+I_{S Y N-}^{V_{d i}}+I_{S Y N \star}^{V_{e I N}} \\
\tau_{b} \frac{d \kappa_{i}}{d t}=-\kappa_{i}+\sigma\left(M_{d i}\right)-\sigma\left(M_{v i}\right)
\end{aligned}
$$

Where, $E_{L}=-60 \mathrm{mV}, E_{C a}=60 \mathrm{mV}, E_{K}=-70 \mathrm{mV}$ are the leak, calcium and potassium reversal potentials respectively. Terms $g_{L}=100 \mathrm{pS}, g_{C a}=400 \mathrm{pS}, g_{K}=500 \mathrm{pS}$ are the maximal leak, calcium and potassium conductances and $C_{m}=3 \mathrm{pF}$ is the membrane capacitance. Timescale parameters were set as $\tau_{n}=30 \mathrm{~ms}, \tau_{u}=85 \mathrm{~ms}, \tau_{b}=10 \mathrm{~ms}$ The function $m_{\infty}(V)=\frac{1}{1+\exp \left(\frac{V_{m}-V}{\theta_{m}}\right)}$ with $\theta_{m}=10.25 \mathrm{mV}, V_{m}=-29 \mathrm{mV}$. And $n_{\infty}(V)=\frac{1}{1+\exp \left(\frac{V_{n}-V}{\theta_{n}}\right)}$ with $\theta_{n}=20 \mathrm{mV}, V_{n}=-55 \mathrm{mV}$. While, $\sigma(V)=\alpha(\tanh (\beta * V)+1)$ with and $\alpha=1000 \mathrm{~mm}^{-1}$ and $\beta=0.01 \mathrm{mV}^{-1}$ converts muscle activities to curvature. The terms $I_{S Y N_{+}}^{V_{d i}} I_{S Y N+}^{V_{\text {elN }}}, I_{S Y N_{-}}^{V_{d i}}, I_{S Y N \star}^{V_{\text {eIN }}}$, respectively are the synaptic currents from the excitatory synapse from eMN to dorsal muscles, excitatory synapse from eINs to the eMN, inhibitory synapse from eMNs to ventral muscles, and the excitatory extrasynaptic input from eIN to ventral muscles. The synaptic currents are based on the graded synapse model used in ${ }^{66}$, and are defined as follows.

$$
\begin{aligned}
& I_{S Y N+}^{V_{d i}}=g\left(V_{d i}\right)\left(M_{d i}-E^{+}\right) \\
& I_{S Y N+}^{V_{\text {eIN }}}=g\left(V_{\text {eIN }}\right)\left(V_{d i}-E^{+}\right) \\
& I_{S Y N-}^{V_{d i}}=g\left(V_{d i}\right)\left(M_{v i}-E^{-}\right) \\
& I_{S Y N \star}^{V_{\text {eIN }}}=g\left(V_{\text {eIN }}\right)\left(M_{v i}-E^{\star}\right) \\
& g(V)=\frac{\bar{g}}{1+\exp \left\{K\left(\frac{V_{k}-V}{V_{\text {range }}}\right)\right\}}
\end{aligned}
$$

With $K=2 \ln \left(\frac{0.1}{0.9}\right)$, set such that the conductance varied from $10 \%$ to $90 \%$ of its maximum value over the presynaptic voltage range set by $V_{\text {range }}$. Other parameters were set as $\bar{g}=1 \mathrm{nS}, V_{k}=$ $-30 \mathrm{mV}, V_{\text {range }}=20 \mathrm{mV}$. Terms $E^{+}, E^{-}, E^{\star}$ are the reversal potentials for the excitatory receptor, inhibitory receptor and the extrasynaptic receptor. We set $E^{+}=-10 \mathrm{mV} . E^{-}$and $E^{\star}$ were treated as variables and optimized to result in an unbiased gait, such that the mean value of the bending curvature $\left\langle\kappa_{i}\right\rangle$ is zero. The optimized values were found to be $E^{-}=-67 \mathrm{mV}, E^{\star}=$ $33.5 \mathrm{mV}$.

\section{MATLAB scripts}

MATLAB scripts developed for behavioral, neuronal and muscle calcium imaging analyses are available in GitHub https://github.com/zhen-lab/Beta_Function_Analysis.

\section{ACKNOWLEDGEMENTS}

We thank Taizo Kawano, Ying Wang, Yan Li, Jin Meng, Sway Chen, Yi Li, Bin Yu, Thomas Sun and Lu Hang for technical support; Albert Cardona for guidance on CATMAID; Michael Koelle and lab members of Mei Zhen, Shangbang Gao and Aravinthan Samuel for discussions. This work was supported by the International Human Frontier Science Program Organization RGP0051/2014 (MZ; ADTS) and the Canadian Institutes of Health Research Foundation Scheme 154274 (MZ).

\section{AUTHOR CONTRIBUTIONS}

$M Z$ and $Q W$ conceived the study; $M Z$ supervised the study; $Y L, T A, A G, B M, W H, J M$ and $M Z$ designed and performed experiments and analyzed data; $D W, B M, D H, M Z$ performed EM reconstruction; AC guided annotation; TA developed model; YL, TA, DW and QW developed scripts; YL, TA, ADTS and MZ wrote the paper; all authors read or edited the paper.

\section{COMPETING INTERESTS}

The authors declare no competing interests. 


\section{References}

[1] Thomas Graham Brown. "Studies in the physiology of the nervous system. XXVIII: Absence of algebraic equality between the magnitudes of central excitation and effective central inhibition given in the reflex centre of a single limb by the same reflex stimulus." In: Quarterly Journal of Experimental Physiology: Translation and Integration 14.1 (1924), pp. 1-23.

[2] Thomas Graham Brown. "The intrinsic factors in the act of progression in the mammal". In: Proceedings of the Royal Society of London. Series B, containing papers of a biological character 84.572 (1911), pp. 308-319.

[3] Ole Kiehn. "Decoding the organization of spinal circuits that control locomotion". In: Nature Reviews Neuroscience 17.4 (2016), p. 224.

[4] Heekyung Jung and Jeremy S Dasen. "Evolution of patterning systems and circuit elements for locomotion". In: Developmental cell 32.4 (2015), pp. 408-422.

[5] Catarina Catela, Maggie M Shin, and Jeremy S Dasen. "Assembly and function of spinal circuits for motor control". In: Annual Review of Cell and Developmental Biology 31 (2015), pp. 669-698.

[6] Neil A Croll. "Components and patterns in the behaviour of the nematode Caenorhabditis elegans". In: Journal of zoology 176.2 (1975), pp. 159-176.

[7] JG White, DG Albertson, and MAR Anness. "Connectivity changes in a class of motoneurone during the development of a nematode". In: Nature 271.5647 (1978), p. 764.

[8] John G White, Eileen Southgate, and J Nichol Thomson. "The structure of the nervous system of the nematode Caenorhabditis elegans". In: (1986).

[9] Janet E Richmond and Erik M Jorgensen. "One GABA and two acetylcholine receptors function at the $\mathrm{C}$. elegans neuromuscular junction". In: Nature neuroscience 2.9 (1999), pp. 791-797.

[10] Shangbang Gao and Mei Zhen. "Action potentials drive body wall muscle contractions in Caenorhabditis elegans". In: Proceedings of the National Academy of Sciences 108.6 (2011), pp. 2557-2562.

[11] Ping Liu et al. "Genetic dissection of ion currents underlying all-or-none action potentials in C. elegans body-wall muscle cells". In: The Journal of physiology 589.1 (2011), pp. 101-117.

[12] Taizo Kawano et al. "An imbalancing act: gap junctions reduce the backward motor circuit activity to bias $\mathrm{C}$. elegans for forward locomotion". In: Neuron 72.4 (2011), pp. 572-586.

[13] John Edward Sulston. "Post-embryonic development in the ventral cord of Caenorhabditis elegans". In: Philosophical Transactions of the Royal Society of London. B, Biological Sciences 275.938 (1976), pp. 287-297.
[14] Eve Marder, Marie L Goeritz, and Adriane G Otopalik. "Robust circuit rhythms in small circuits arise from variable circuit components and mechanisms". In: Current opinion in neurobiology 31 (2015), pp. 156-163.

[15] John E Sulston and H Robert Horvitz. "Post-embryonic cell lineages of the nematode, Caenorhabditis elegans". In: Developmental biology 56.1 (1977), pp. 110-156.

[16] Martin Chalfie et al. "The neural circuit for touch sensitivity in Caenorhabditis elegans". In: Journal of Neuroscience 5.4 (1985), pp. 956-964.

[17] Nathan C Klapoetke et al. "Independent optical excitation of distinct neural populations". In: Nature methods 11.3 (2014), pp. 338-346.

[18] Elena G Govorunova et al. "Natural light-gated anion channels: A family of microbial rhodopsins for advanced optogenetics". In: Science 349.6248 (2015), pp. 647-650.

[19] Yehezkel Ben-Ari. "Excitatory actions of gaba during development: the nature of the nurture". In: Nature Reviews Neuroscience 3.9 (2002), p. 728.

[20] Bingjie Han, Andrew Bellemer, and Michael R Koelle. "An evolutionarily conserved switch in response to GABA affects development and behavior of the locomotor circuit of Caenorhabditis elegans". In: Genetics 199.4 (2015), pp. 1159-1172.

[21] Jana F Liewald et al. "Optogenetic analysis of synaptic function”. In: Nature methods 5.10 (2008), pp. 895-902.

[22] Mei Zhen and Aravinthan DT Samuel. "C. elegans locomotion: small circuits, complex functions". In: Current opinion in neurobiology 33 (2015), pp. 117-126.

[23] Yingchuan B Qi et al. "Photo-inducible cell ablation in Caenorhabditis elegans using the genetically encoded singlet oxygen generating protein miniSOG”. In: Proceedings of the National Academy of Sciences 109.19 (2012), pp. 7499-7504.

[24] Christian Schultheis et al. "Optogenetic analysis of GABAB receptor signaling in Caenorhabditis elegans motor neurons". In: Journal of neurophysiology 106.2 (2011), pp. 817-827.

[25] Yu Shen et al. "An extrasynaptic GABAergic signal modulates a pattern of forward movement in Caenorhabditis elegans". In: Elife 5 (2016), e14197.

[26] Jeremy S Dittman and Joshua M Kaplan. "Behavioral impact of neurotransmitter-activated G-protein-coupled receptors: muscarinic and $\mathrm{GABAB}$ receptors regulate Caenorhabditis elegans locomotion". In: Journal of Neuroscience 28.28 (2008), pp. 7104-7112.

[27] Christelle Gally and Jean-Louis Bessereau. "GABA is dispensable for the formation of junctional GABA receptor clusters in Caenorhabditis elegans". In: Journal of Neuroscience 23.7 (2003), pp. 2591-2599. 
[28] Berangere Pinan-Lucarre et al. "C. elegans Punctin specifies cholinergic versus GABAergic identity of postsynaptic domains". In: Nature 511.7510 (2014), pp. 466-470.

[29] Laura Pereira et al. "A cellular and regulatory map of the cholinergic nervous system of C. elegans". In: Elife 4 (2015), e12432.

[30] Shangbang Gao et al. "Excitatory motor neurons are local oscillators for backward locomotion". In: Elife 7 (2018), e29915.

[31] Tianqi $\mathrm{Xu}$ et al. "Descending pathway facilitates undulatory wave propagation in Caenorhabditis elegans through gap junctions". In: Proceedings of the National Academy of Sciences 115.19 (2018), E4493-E4502.

[32] Sean T Sweeney et al. "Targeted expression of tetanus toxin light chain in Drosophila specifically eliminates synaptic transmission and causes behavioral defects". In: Neuron 14.2 (1995), pp. 341-351.

[33] Jordan Hylke Boyle, Stefano Berri, and Netta Cohen. "Gait modulation in C. elegans: an integrated neuromechanical model". In: Frontiers in computational neuroscience 6 (2012), p. 10.

[34] Akira Sakurai and Paul S Katz. "Artificial synaptic rewiring demonstrates that distinct neural circuit configurations underlie homologous behaviors". In: Current Biology 27.12 (2017), pp. 1721-1734.

[35] Timothy O'Leary and Eve Marder. "Temperature-robust neural function from activity-dependent ion channel regulation". In: Current Biology 26.21 (2016), pp. 2935-2941.

[36] Citlali Trueta and Francisco Fernandez De-Miguel. "Extrasynaptic exocytosis and its mechanisms: a source of molecules mediating volume transmission in the nervous system". In: Frontiers in physiology 3 (2012), p. 319 .

[37] Meghan A Jobson et al. "Spillover transmission is mediated by the excitatory GABA receptor LGC-35 in C. elegans". In: Journal of Neuroscience 35.6 (2015), pp. 2803-2816.

[38] Francisco Fernandez De-Miguel and Kjell Fuxe. "Extrasynaptic neurotransmission as a way of modulating neuronal functions". In: Frontiers in physiology 3 (2012), p. 16

[39] Michele Zoli et al. "The emergence of the volume transmission concept". In: Brain Research Reviews 26.2-3 (1998), pp. 136-147.

[40] ES Vizi et al. "Non-synaptic receptors and transporters involved in brain functions and targets of drug treatment". In: British journal of pharmacology 160.4 (2010), pp. 785-809.

[41] Eve Marder. "Neuromodulation of neuronal circuits: back to the future". In: Neuron 76.1 (2012), pp. 1-11.

[42] Barry Bentley et al. "The multilayer connectome of Caenorhabditis elegans". In: PLoS computational biology 12.12 (2016), e1005283.
[43] Peter A Getting. "Emerging principles governing the operation of neural networks". In: Annual review of neuroscience 12.1 (1989), pp. 185-204.

[44] Kevin L Briggman and WB Kristan Jr. "Multifunctional pattern-generating circuits". In: Annu. Rev. Neurosci. 31 (2008), pp. 271-294.

[45] Anthony D Fouad et al. "Distributed rhythm generators underlie Caenorhabditis elegans forward locomotion". In: Elife 7 (2018), e29913.

[46] Didier Combes et al. "Multiple ace genes encoding acetylcholinesterases of Caenorhabditis elegans have distinct tissue expression". In: European Journal of Neuroscience 18.3 (2003), pp. 497-512.

[47] Jordan H Boyle and Netta Cohen. "A minimal model of C. elegans forward locomotion: the larval L1 circuit". In: BMC Neuroscience 12.1 (2011), pp. 1-1.

[48] Julijana Gjorgjieva, David Biron, and Gal Haspel. "Neurobiology of Caenorhabditis elegans locomotion: where do we stand?" In: Bioscience 64.6 (2014), pp. 476-486.

[49] Alejandro Bilbao et al. "Roll maneuvers are essential for active reorientation of Caenorhabditis elegans in 3D media". In: Proceedings of the National Academy of Sciences 115.16 (2018), E3616-E3625.

[50] Ben Mulcahy et al. "A pipeline for volume electron microscopy of the Caenorhabditis elegans nervous system". In: Frontiers in neural circuits 12 (2018), p. 94.

[51] Daniel Witvliet et al. "Connectomes across development reveal principles of brain maturation". In: Nature (2021), pp. 1-5.

[52] Albert Cardona et al. "TrakEM2 software for neural circuit reconstruction". In: PloS one 7.6 (2012), e38011.

[53] Johannes Schindelin et al. "Fiji: an open-source platform for biological-image analysis". In: Nature methods 9.7 (2012), pp. 676-682.

[54] Stephan Saalfeld et al. "CATMAID: collaborative annotation toolkit for massive amounts of image data". In: Bioinformatics 25.15 (2009), pp. 1984-1986.

[55] Georg Nagel et al. "Light activation of channelrhodopsin-2 in excitable cells of Caenorhabditis elegans triggers rapid behavioral responses". In: Current Biology 15.24 (2005), pp. 2279-2284.

[56] Maria A Lim et al. "Neuroendocrine modulation sustains the C. elegans forward motor state". In: Elife 5 (2016), e19887.

[57] Quan Wen et al. "Proprioceptive coupling within motor neurons drives C. elegans forward locomotion". In: Neuron 76.4 (2012), pp. 750-761.

[58] Zengcai V Guo, Anne C Hart, and Sharad Ramanathan. "Optical interrogation of neural circuits in Caenorhabditis elegans". In: Nature methods 6.12 (2009), p. 891.

[59] John Peter Rickgauer, Karl Deisseroth, and David W Tank. "Simultaneous cellular-resolution optical perturbation and imaging of place cell firing fields". In: Nature neuroscience 17.12 (2014), p. 1816. 
[60] Jasper Akerboom et al. "Genetically encoded calcium indicators for multi-color neural activity imaging and combination with optogenetics". In: Frontiers in molecular neuroscience 6 (2013), p. 2.

[61] Christina K Kim et al. "Simultaneous fast measurement of circuit dynamics at multiple sites across the mammalian brain". In: Nature methods 13.4 (2016), p. 325 .

[62] Adam M Packer et al. "Simultaneous all-optical manipulation and recording of neural circuit activity with cellular resolution in vivo". In: Nature methods 12.2 (2015), p. 140.

[63] Priyamvada Rajasethupathy et al. "Projections from neocortex mediate top-down control of memory retrieval". In: Nature 526.7575 (2015), p. 653.

[64] Zihui Zhang et al. "Closed-loop all-optical interrogation of neural circuits in vivo". In: Nature methods 15.12 (2018), pp. 1037-1040.

[65] Catherine Morris and Harold Lecar. "Voltage oscillations in the barnacle giant muscle fiber". In: Biophysical journal 35.1 (1981), pp. 193-213.

[66] Stephen R Wicks, Chris J Roehrig, and Catharine H Rankin. "A dynamic network simulation of the nematode tap withdrawal circuit: predictions concerning synaptic function using behavioral criteria". In: Journal of Neuroscience 16.12 (1996), pp. 4017-4031.

[67] Cornelia Schmitt et al. "Specific expression of channelrhodopsin-2 in single neurons of Caenorhabditis elegans". In: PloS one 7.8 (2012).

[68] Victoria J Butler et al. "A consistent muscle activation strategy underlies crawling and swimming in Caenorhabditis elegans". In: Journal of the Royal Society Interface 12.102 (2015), p. 20140963. 
bioRxiv preprint doi: https://doi.org/10.1101/2021.09.21.461278; this version posted December 21, 2021. The copyright holder for this preprint (which was not certified by peer review) is the author/funder, who has granted bioRxiv a license to display the preprint in perpetuity. It is made available under aCC-BY-NC-ND 4.0 International license.

\section{Supplemental figures}
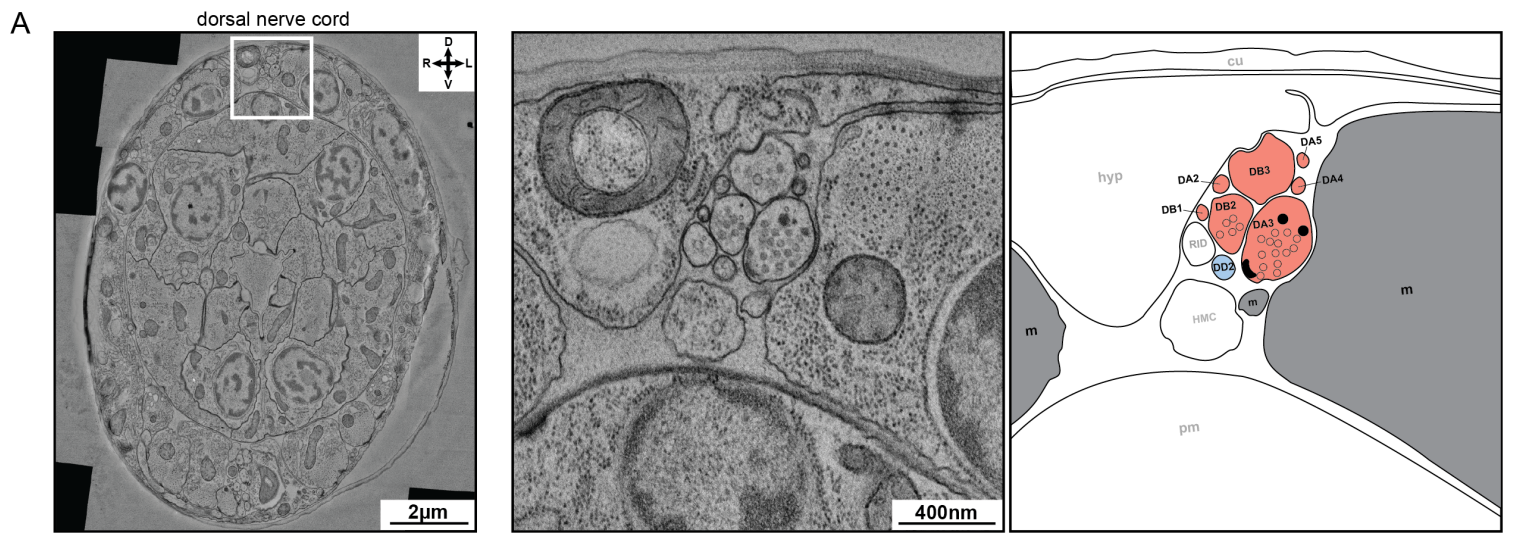

B
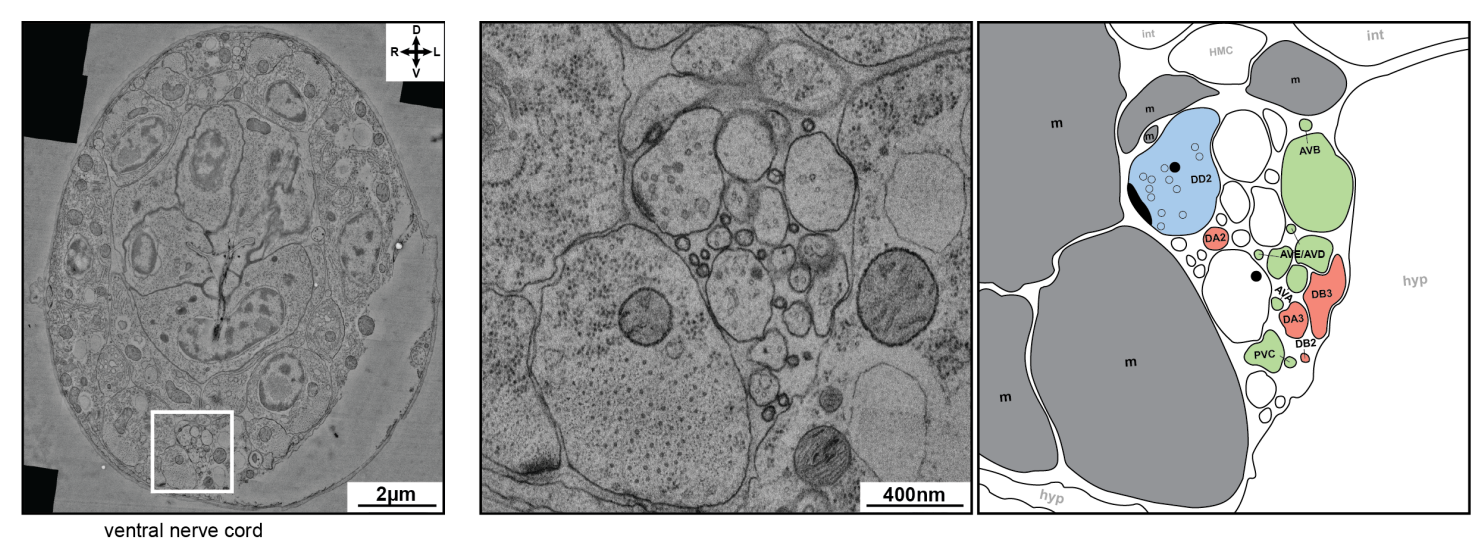

Figure S1. Electron microscopy reconstruction of the dorsal and ventral nerve cord of an 5-6hr L1 larva. A. Example image of the dorsal nerve cord. (left) An electron micrograph of the cross-section in transverse plane. The dorsal nerve cord is marked. (center) An enlarged view of the dorsal nerve cord. right Cross-sections of individual neurites of motor neurons and muscles of the L1 motor circuit were color-coded and annotated. Red: eMNs; blue:iMNs; grey: muscles. One eMN (DA3) makes a dyadic NMJ to the muscle and iMN (DD2), with an active zone (black crescent), a cluster of synaptic vesicles (clear circles) and sparse dense core vesicles (dark circles). B. Example image of the ventral nerve cord, similarly illustrated and annotated as in A. Neurites for elNs, which are absent from the dorsal nerve cord (A), are color-coded green. An iMN (DD2) makes a NMJ to muscles. In right panels, cross-sections of other neuron (RID) and cell (HMC) of the L1 motor circuit in the dorsal nerve cord (A) and ventral nerve cord (B) were labelled by opaque letters. Abbreviations: m: muscle; pm: pharyngeal muscle; hyp: hypodermis; cu: cuticle; int: intestine. 
bioRxiv preprint doi: https://doi.org/10.1101/2021.09.21.461278; this version posted December 21, 2021. The copyright holder for this preprint (which was not certified by peer review) is the author/funder, who has granted bioRxiv a license to display the preprint in perpetuity. It is made available under aCC-BY-NC-ND 4.0 International license.

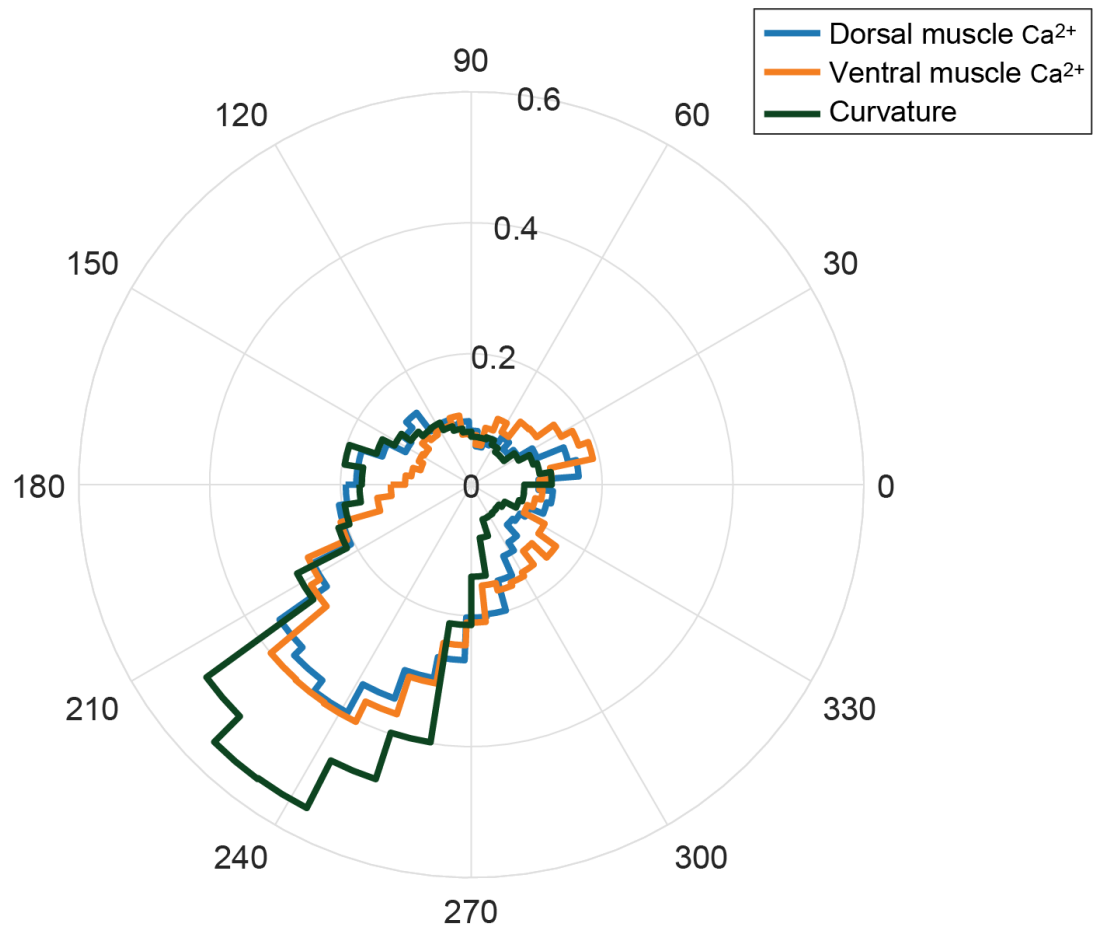

Figure S2. Polar histograms of phase lags of muscle activity and of curvature between segments along the body. L1 larvae crawled slowly under this imaging condition, and phase differences are similar for calcium and bending waves. 
bioRxiv preprint doi: https://doi.org/10.1101/2021.09.21.461278; this version posted December 21, 2021. The copyright holder for this preprint (which was not certified by peer review) is the author/funder, who has granted bioRxiv a license to display the preprint in perpetuity. It is made available under aCC-BY-NC-ND 4.0 International license.

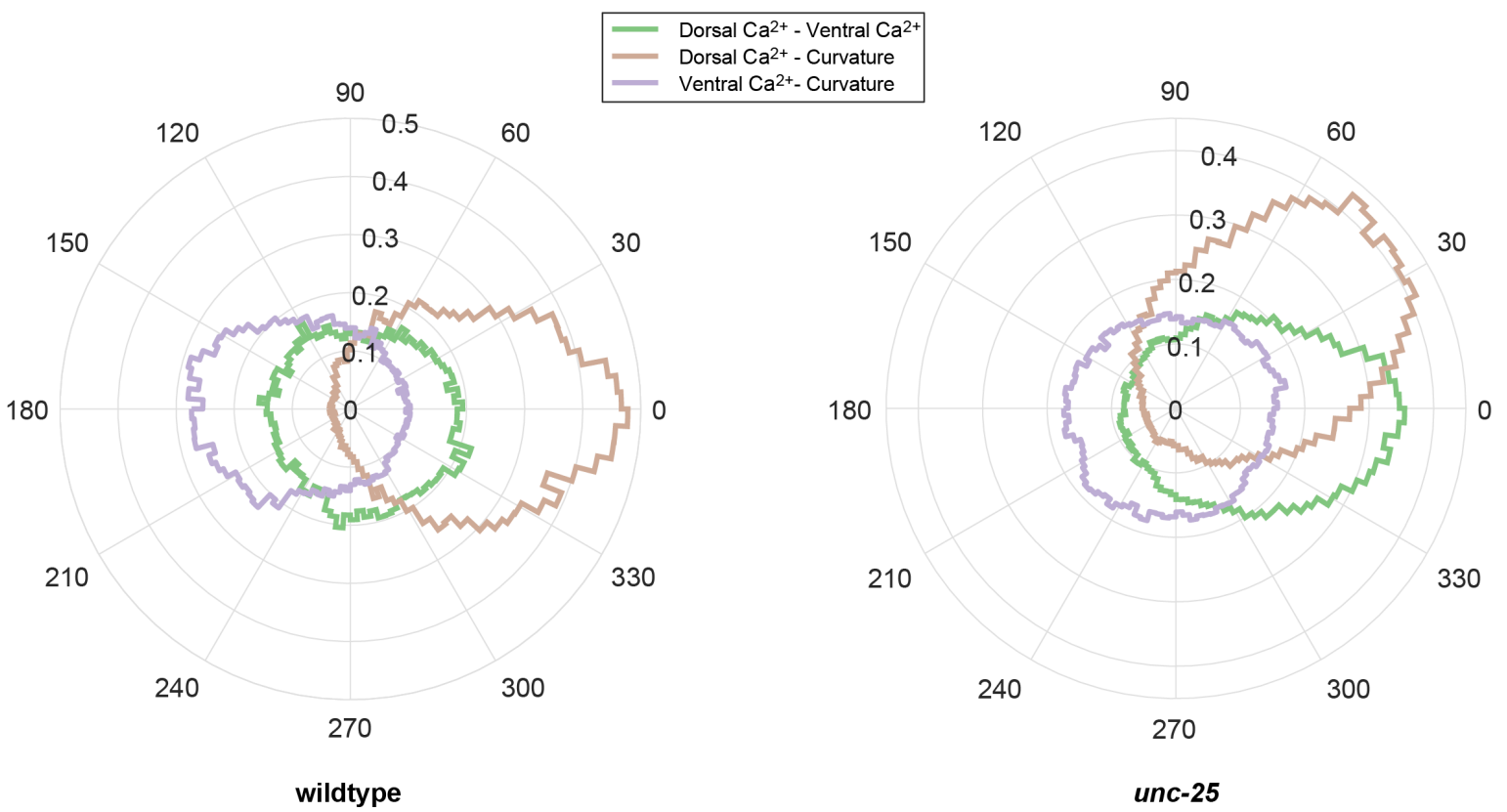

Figure S3. Calcium dynamics of dorsal muscles tracks bend waves in unc-25 mutant larvae. Polar histograms of the phase differences between curvature, dorsal muscle activity, and ventral muscle activity (segments 33-95) for crawling wildtype and unc-25 larvae imaged under the same condition. left In wildtype larvae, dorsal muscle activity was in phase with body bending and ventral muscle was antiphasic with body bending. right In unc-25 larvae, ventral muscles had no phase relationship with curvature, but dorsal muscle activity tracked body bending with phase lag. 


\section{A}

\section{DB ablated}
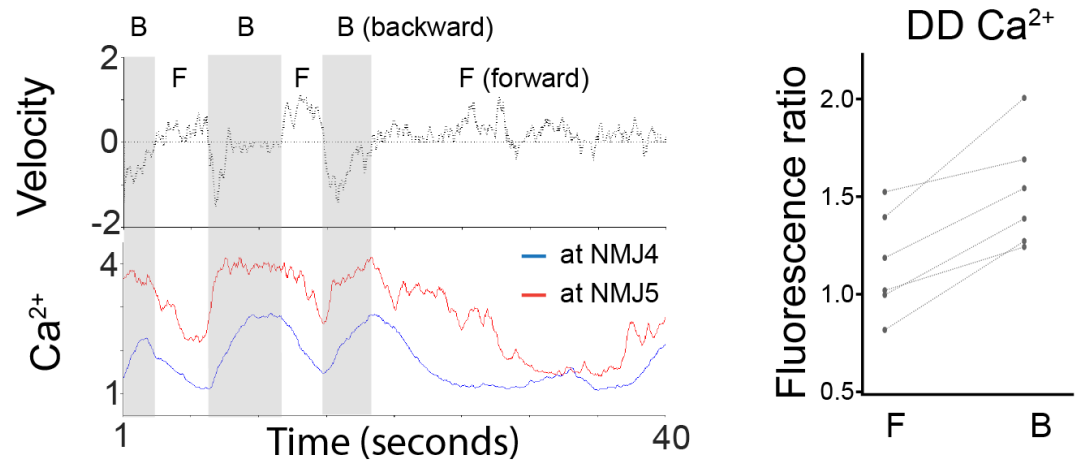

B
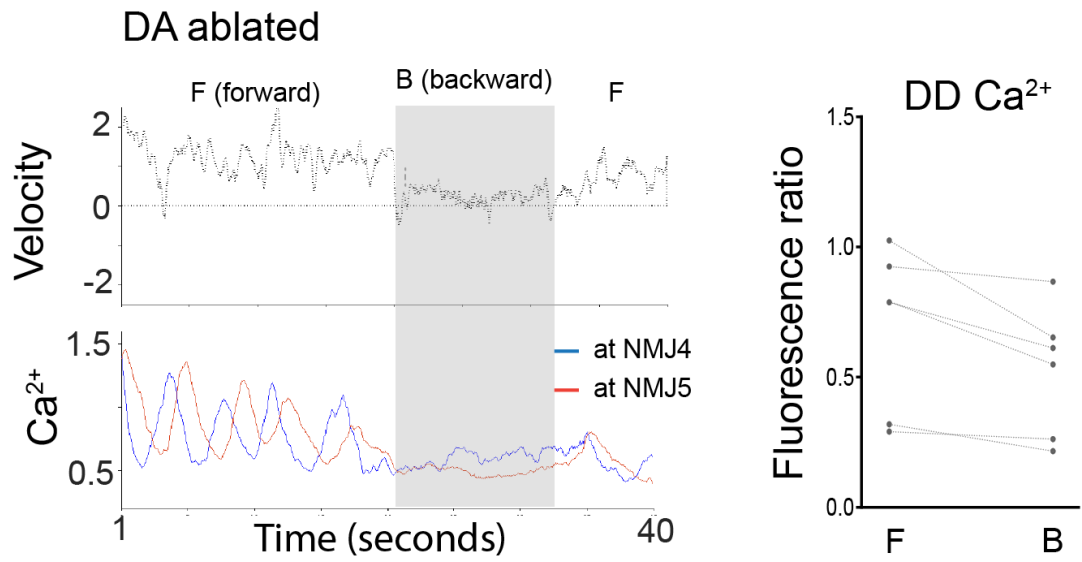

Figure S4. Ablation of eMNs reduces iMN activity during directional movement. A. (left) Example of velocity and DD NMJ calcium of an L1 lava upon DA motor neuron ablation. This larva still transited between forward and backward movement, with attempted reversals shown as pauses. (right) Mean DD activity during forward and backward movement. Dotted lines represented epochs from the same animal. B. (left) Example of velocity and DD NMJ calcium of an L1 lava upon DB motor neuron ablation. This larva transited between forward and backward movement, with attempted forward movement shown as pauses. (right) Mean DD activity during forward and backward movement, with lines connecting epochs from the same animal. 
bioRxiv preprint doi: https://doi. org/101101/2021.09.21.461278. this version posted December 21, 2021. The copyright holder for this preprint (which was not certified by peer review) is the author/funder, who has granted bioRxiv a license to display the preprint in perpetuity. It is made available under aCC-BY-NC-ND 4.0 International license.

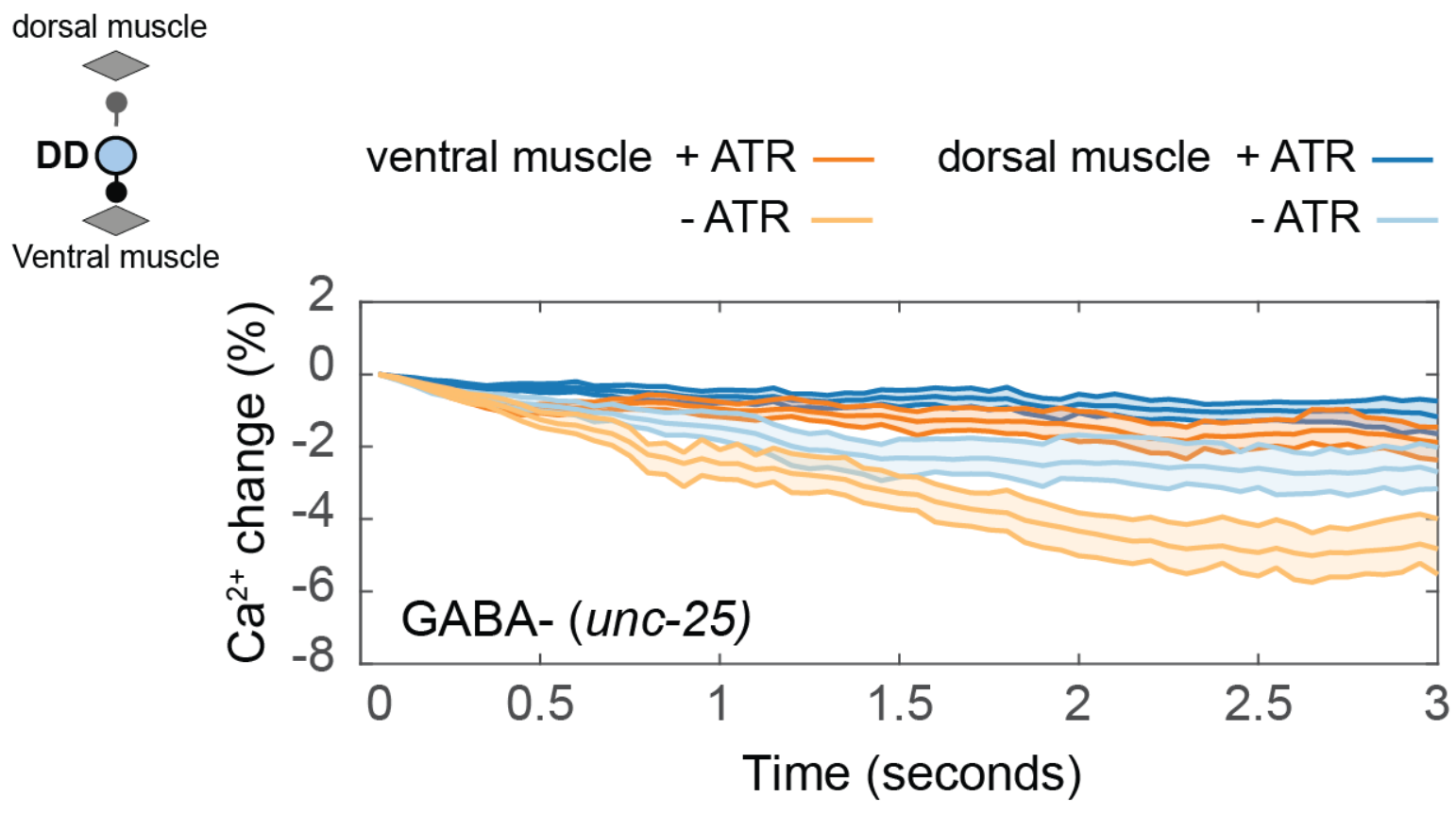

Figure S5. Activation of iMNs does not significantly reduce ventral and dorsal muscle activity in mutant larvae that do not synthesize GABA. (inset) Schematic of simultaneous optogenetic activation of iMNs and muscle calcium imaging. Y-axis plots percentage changes of the muscle activity from $t=0$. Control group (-ATR): 34 stimulation epochs from 12 larvae; Experimental group (+ATR): 15 stimulation epochs from 5 larvae. 
bioRxiv preprint doi: https://doi.org/10.1101/2021.09.21.461278; this version posted December 21, 2021. The copyright holder for this preprint (which was not certified by peer review) is the author/funder, who has granted bioRxiv a license to display the preprint in perpetuity. It is made available under aCC-BY-NC-ND 4.0 International license.

A

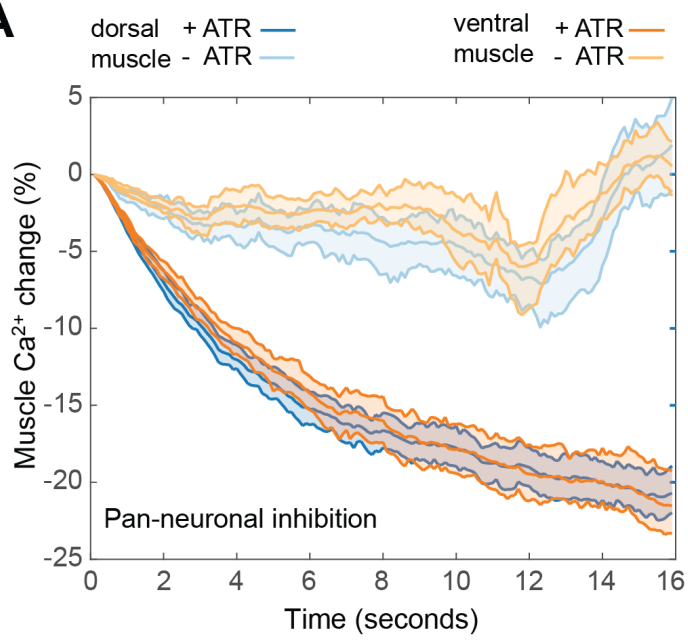

C

dorsal + ATR muscle - ATR

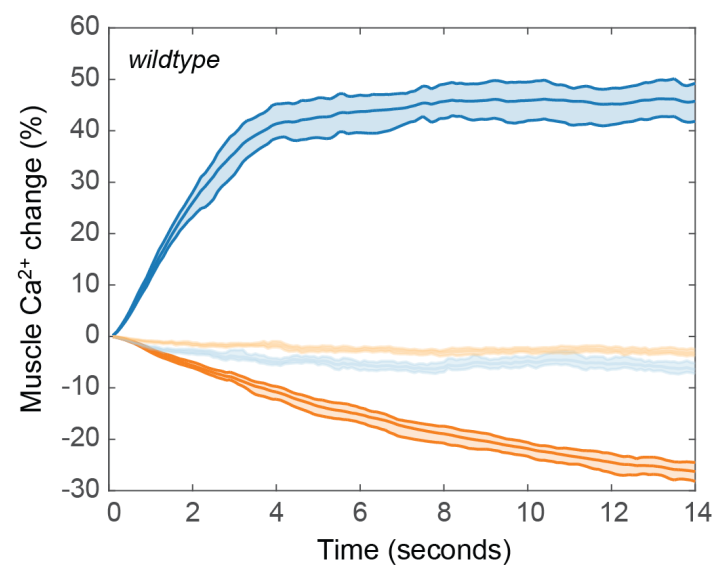

D Pan-cholinergic neuronal activation dorsal + ATR muscle - ATR -

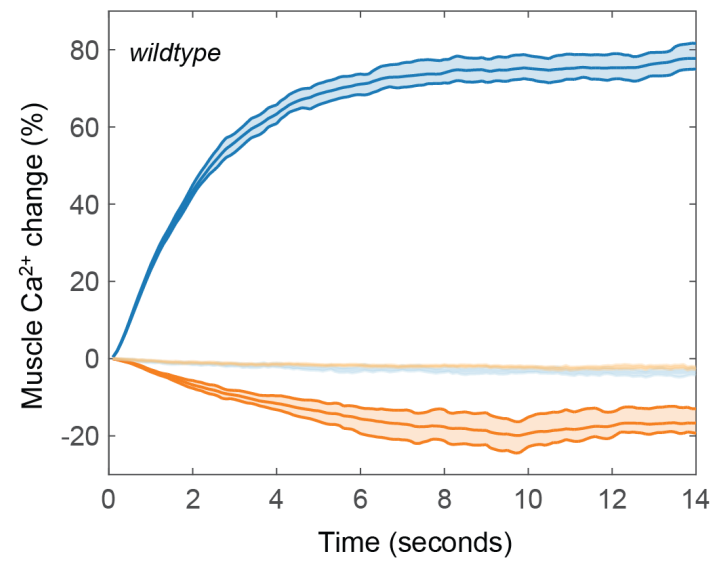

B

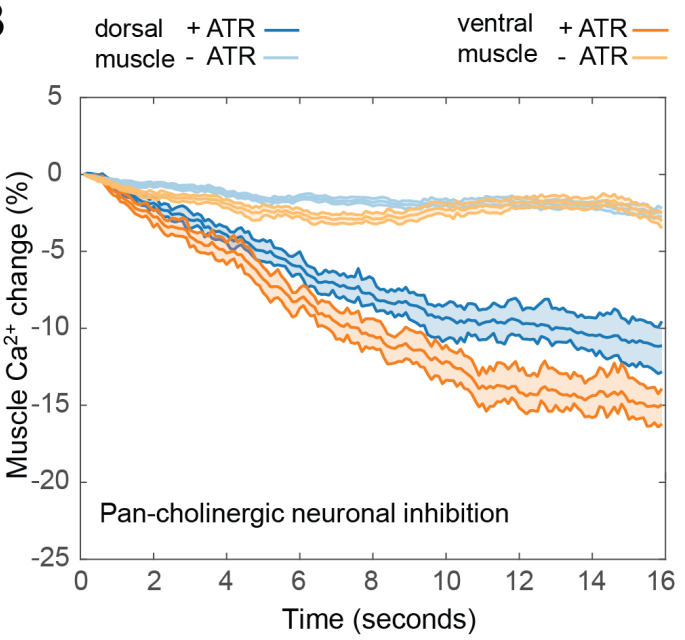

ventral + ATR -

muscle - ATR

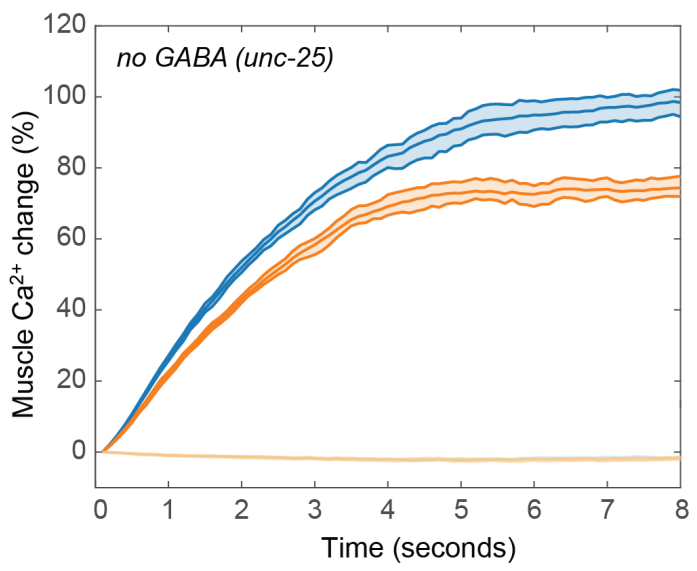

ventral + ATR -

muscle - ATR -

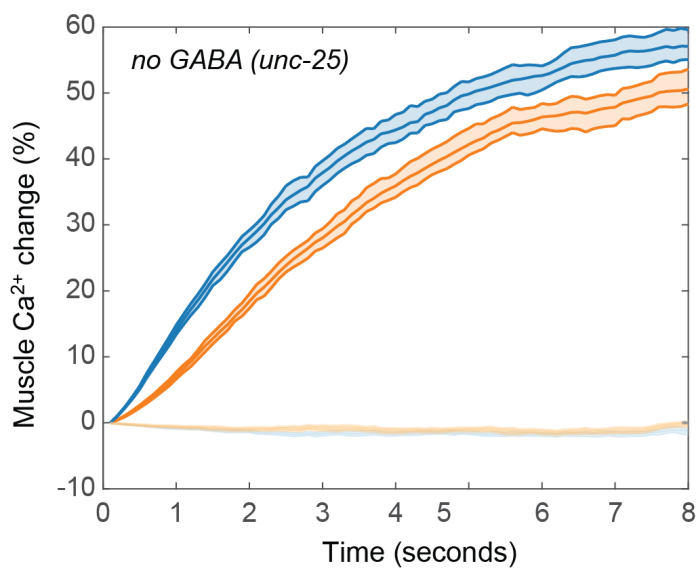

Figure S6. Effect of optogenetic manipulation of groups of neurons on dorsal and ventral muscles A, B Muscle calcium responses upon optogenetic silencing of all neurons (A) or all cholinergic neurons (B) in wildtype larvae. Y-axis plots percentage changes of the muscle activity from $t=0$. Panneuronal inhibition: the control group (-ATR): 18 stimulation epochs from 6 larvae; the experimental group (+ATR): 26 stimulation epochs from 12 larvae. Cholinergic neuron inhibition: the control group (-ATR), 16 stimulation epochs from 3 larvae; the experimental group (+ATR), 27 stimulation epochs from 5 larvae. C. Muscle calcium responses upon optogenetic activation of all neurons in wildtype (left) or unc-25 (right) larvae. wildtype group: -ATR: 17 stimulation epochs from 9 larvae; +ATR: 18 stimulation epochs from 10 larvae. unc-25 group: -ATR: 14 stimulation epochs from 9 larvae; +ATR: 21 stimulation epochs from 11 larvae. D. Muscle calcium responses upon pan-cholinergic activation in wildtype (left) or unc-25 (right) larvae. wildtype group: -ATR, 15 stimulation epochs from 7 larvae; +ATR, 19 stimulation epochs from 10 larvae. unc-25 group: -ATR, 18 stimulation epochs from 6 larvae; +ATR, 30 stimulation epochs from 10 larvae. 
A

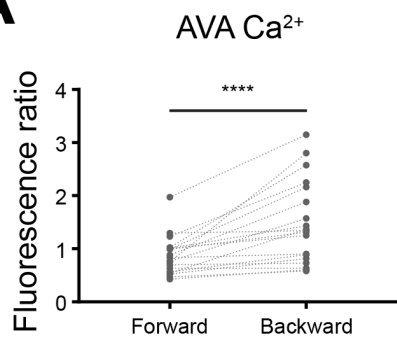

C

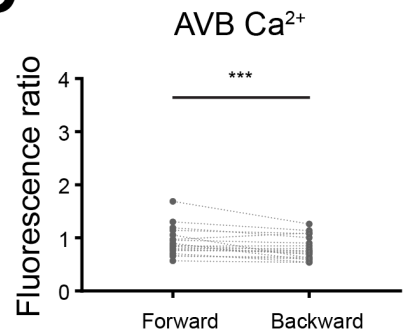

B

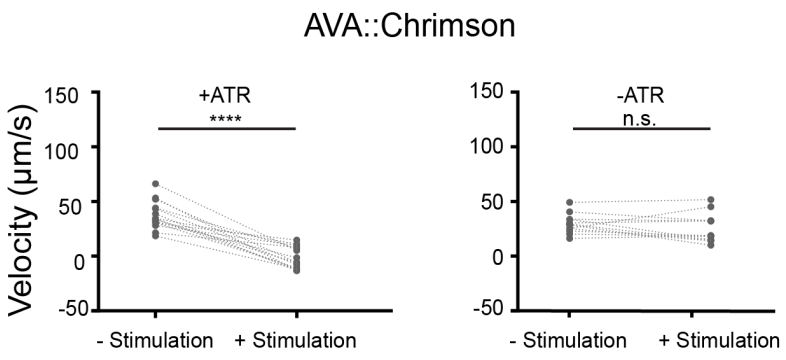

D

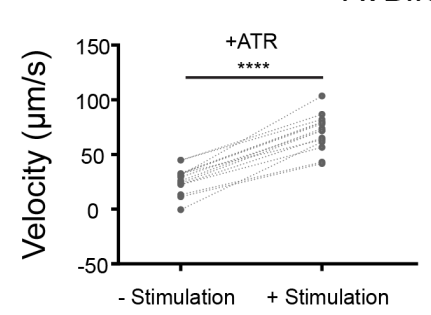

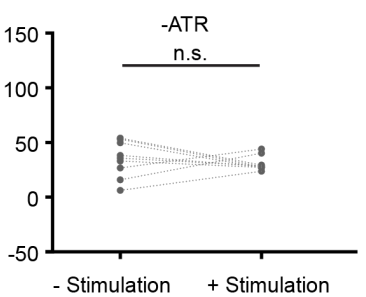

Figure S7. Subgroups of elNs potentiate directional movements in L1 larvae. A. The AVA elN exhibited higher activity during backward movement. $n=19$ larvae. B. Optogenetic activation of AVA led to reversals. Mean velocities during non-stimulated and stimulated phases were compared in the control group (-ATR) ( $\mathrm{n}=12$ larvae) and the experimental group $(+A T R)\left(n=15\right.$ larvae). ${ }^{* * * *} p<0.0001$. C. The AVB elN exhibited higher activity during forward movement $\left(n=19\right.$ larvae). ${ }^{* * *} p=0.0006$. D. Optogenetic activation of AVB led to increased forward velocity. Control group (- ATR): $n=9$ larvae. Experimental group $(+A T R): n=15$ larvae. ${ }^{\star \star \star \star} p<0.0001$. All $p$-values were calculated using the Wilcoxon matched-pairs signed rank test. 


\section{Movie captions}

Supplemental Movie 1 (Fig. 1C): L1 larvae generate alternating dorsal and ventral body bends

a) An L1 larva swimming in M9 solution. In the first frame, head is up and tail is down. A lump of bacteria food anchored this happy baby. b) An L1 larva crawling on a NGM plate with a thin layer of bacteria food. In the first frame, head is down, tail is up, dorsal is left and ventral is right.

Supplemental Movie 2 (Fig. 1D): L1 larvae exhibit phaselagged, curvature-correlated dorsal and ventral muscle calcium dynamics

Upper panels Calcium imaging of a crawling L1 larva that expressed GCaMP3::RFP in boy wall muscles. Head: left, tail: right, dorsal: up, and ventral: down. GCaMP3/RFP ratio was segmented along the body. Color bar and the Y-axis of the lower panel have the same scale. Dashed line denotes the segment that separates the head and body. Lower panel Calcium signals in dorsal (blue) and ventral (red) muscles over time. X-axis represents body segments (head: 1, tail: 100); dashed line denotes the segment that separates the head and body. Y-axis represents the GCaMP3/RFP ratio at each segment.

Supplemental Movie 3 (Fig. 2A): Sequential increase of DA motor neuron activity during L1 larva's backward movement.

Calcium imaging of a crawling L1 larva expressing GCaMP6::RFP in the DA subclass of eMNs. Head: left; tail: right; dorsal: up; and ventral: down. GCaMP6 and RFP signals from DA3-DA9 somata were tracked. Left-pointing arrows denote periods of forward movement; right-point arrows periods of backward movement. Grey bars denote periods of backward movement.

Supplemental Movie 4 (Fig. 2B): Sequential increase of DB motor neuron activity during L1 larva's forward movement.

Calcium imaging of a crawling L1 larva expressing GCaMP6::RFP in DA and DB motor neurons. Head: left; tail: right; dorsal: up; ventral: down. GCaMP6 and RFP signals from DB4-DB6 somata were tracked. Left-pointing arrows denote periods of forward movement; right-point arrows periods of backward movement. Grey bars denote periods of forward movement.

Supplemental Movie 5 (Fig. 3A): NMJs from iMNs are activated during both forward and backward movements.

Calcium imaging of a crawling L1 larva expressing GCaMP6::RFP in DD motor neurons. Head: left; tail: right; dorsal: up; ventral: down. GCaMP6 and RFP signals from NMJs from DD5 and DD6 were tracked. Left-pointing arrows denote periods of forward movement; right-point arrows periods of backward movement. Grey bars denote periods of backward movement.
Supplemental Movie 6 (Fig. 3D, E): Activation of iMNs elevates dorsal and ventral muscle activity with different spatial patterns

Upper panels Calcium imaging of an immobilized L1 larva that expressed GCaMP6::RFP in muscle cells and chrimson in DD motor neurons. Head: left, tail: right, dorsal: up and ventral: down. Dashed line denotes the body segment that separates the head and the body. Lower panel Calcium signals in dorsal (blue) and ventral (red) muscles over time. X-axis represents body segments (head: 1; tail: 100); the vertical dashed line denotes the segment that separates the head and body. Y-axis represents GCaMP6/RFP value at each segment.

Supplemental Movie 7 (Fig. 3F; Fig. 3S): Calcium signals in dorsal and ventral muscles exhibit distinct patterns in crawling unc-25 mutant larvae.

Upper panels Calcium imaging of a crawling unc-25 mutant larva that expressed GCaMP3::RFP in muscle cells. Head: left, tail: right, dorsal: up; ventral: down. Color bar and Y-axis of the lower panel represent the same scale. Dashed line denotes the body segment that separates the head and the body. Lower panel Calcium signals in dorsal (blue) and ventral (red) muscles over time. X-axis represents body segments (head: 1; tail: 100); the vertical dashed line denotes the segment that separates the head and body. Y-axis represents GCaMP3/RFP value at each segment.

\section{Supplemental Movie 8a (Fig. 4): iMNs are activated upon stimulation of all eMNs.}

Calcium imaging of an immobilized L1 larva that expressed GCaMP6::RFP in DD motor neurons and chrimson in DA and DB motor neurons. Head: left, tail: right, dorsal: up and ventral: down. NMJs from DD4 and DD5 were tracked. Right panel shows percentage changes of the muscle activity from $t=0$.

\section{Supplemental Movie 8b (Fig.4): iMNs are inactivated upon eMN silencing.}

Calcium imaging of an immobilized L1 larva that expressed GCaMP6::RFP in DD motor neuorns and GtACR2 in DA and DB motor neurons. Head: left, tail: right, dorsal: up and ventral: down. NMJs from DD5, DD6 and DD7 were tracked. Right panel shows percentage changes of the muscle activity from $t=0$.

Supplemental Movie 9 (Fig. 6A): Activation of elNs induces spatially distinct calcium increase in unc-25 mutant larva's dorsal and ventral muscles

Upper panels Calcium imaging of an immobilized unc-25 mutant larva that expressed GCaMP3::RFP in body wall muscles and ChR2 in AVA. Head: left, tail: right, dorsal: up and ventral: down. Color bar and Y-axis of the lower panel represent the same scale. Dashed line denotes the body segment that separates the head and the body. Lower panel Calcium signals in dorsal (blue) and ventral (red) muscles over time. X-axis represents body segments (head: 1 ; tail: 100); the vertical dashed line denotes the segment that separates the head and body. Y-axis represents GCaMP3/RFP value at each segment. 


\section{Supplemental Movie 10 (Fig. 6B): Silencing elNs reduces both dorsal and ventral muscle activities.}

Upper panels Calcium imaging of an immobilized L1 larva that expressed GCaMP3::RFP in body wall muscles and chrimson in multiple cholinergic premotor interneurons. Head: left, tail: right, dorsal: up and ventral: down. Color bar and Y-axis of the lower panel represent the same scale. Dashed line denotes the body segment that separates the head and the body. Lower panel Calcium signals in dorsal (blue) and ventral (red) muscles over time. X-axis represents body segments (head: 1 ; tail: 100); the vertical dashed line denotes the segment that separates the head and body. Y-axis represents GCaMP3/RFP value at each segment.

Supplemental Movie 11 (Fig. 7A): Calcium signal in ventral muscles is reduced after ablation of elNs in wildtype larvae.

Upper panels Calcium imaging of an unconstrained L1 larva that expressed GCaMP3::RFP in body wall muscles and miniSOG in all eINs after photo-ablation. It adopted a dorsally biased posture. GCaMP3/RFP ratio was segmented along the body. Color bar and Y-axis of the lower panel represent the same scale. Dashed line denotes the body segment that separates the head and the body. Lower panel Calcium signals in dorsal (blue) and ventral (red) muscles over time. X-axis represents body segments (head: 1; tail: 100); the vertical dashed line denotes the segment that separates the head and body. Y-axis represents GCaMP3/RFP value at each segment.

\section{Supplemental Movie 12 (Fig. 7B): Calcium signal in ventral muscles is reduced after eIN ablation in unc-25(e156) mutant larvae.}

Upper panels Calcium imaging of an unconstrained L1 unc-25 larva that expressed GCaMP3::RFP in body wall muscles and miniSOG in all eINs after photo-ablation. It adopted a dorsally biased posture. Color bar and Y-axis of the lower panel represent the same scale. Dashed line denotes the body segment that separates the head and the body. Lower panel Calcium signals in dorsal (blue) and ventral (red) muscles over time. X-axis represents body segments (head: 1 ; tail: 100); the vertical dashed line denotes the segment that separates the head and body. Y-axis represents GCaMP3/RFP value at each segment.

Supplemental Movie 13 (Fig. 7C): Calcium signal in ventral muscles is reduced when synaptic vesicle release from elNs is blocked.

Upper panels Calcium imaging of an uncontrained L1 larva that expressed GCaMP3::RFP in body wall muscles and TeTx in all eINs. It crawled with a dorsally biased posture. Color bar and Y-axis of the lower panel represent the same scale. Dashed line denotes the body segment that separates the head and the body. Lower panel Calcium signals in dorsal (blue) and ventral (red) muscles over time. X-axis represents body segments (head: 1; tail: 100); the vertical dashed line denotes the segment that separates the head and body. Y-axis represents GCaMP3/RFP value at each segment.
Supplemental Movie 14 (Fig. 7D): Blocking synaptic vesicle release from eMNs reveals uniformed calcium signals in ventral muscles of L1 larvae.

Upper panels Calcium imaging of an unconstrained L1 larva that expressed GCaMP3::RFP in body wall muscles and TeTx in eMNs. GCaMP3/RFP ratio was segmented along the body. Color bar and Y-axis of the lower panel represent the same scale. The vertical dashed line denotes the body segment that separates the head and the body. Lower panel Calcium signals in dorsal (blue) and ventral (red) muscles over time. X-axis represents body segments (head: 1; tail: 100); the vertical dashed line denotes the segment that separates the head and body. Y-axis represents GCaMP3/RFP value at each segment.

Supplemental Movie 15 (Discussion): Complex bending defects of unc-25 mutant larvae

unc-25 mutant larvae exhibit bending defects that are difficult to quality for ventral or dorsal bias. Qualitatively, they rest in a body posture with ventral bending across the body. When they are prompted to move, their forward movement does not exhibit overt dorsal or ventral bias, whereas their reversals include rapid ventral coils. Same trends are observed for crawling and swimming. 


\section{Constructs and Strains}

Transgenic ZM strains were generated by injecting fosmid or plasmid constructs (pJH) at 2-50ng/ul, with respective injection markers into indicated genetic backgrounds, to produce those with extra chromosomal arrays (hpEx). They were integrated into the genome by UV irradiation of the Ex lines followed by selective screening and outcrossing against N2 wild-type strains (hpIs). Other transgenic arrays or strains were acquired from either $C G C$ or individual laboratories.

Table S1. Constructs and Strains information

\begin{tabular}{|c|c|c|c|}
\hline Strain & Genotype & Construct & Notes \\
\hline $\mathrm{N} 2$ & wild-type & & \\
\hline CB156 & $u n c-25(e 156) \mathrm{III}$ & & without GABA \\
\hline CB407 & unc-49(e407) III & & without GABAa \\
\hline ZM9062 & hpIs583 [Pacr-2s-miniSOG::UrSL2::wCherry + lin-15(+)] & pJH3626 & iMN ablation \\
\hline ZM9062 & hpIs583 [Pacr-2s-miniSOG::SL2::RFP+lin-15(+)] & pJH3626 & eMN ablation \\
\hline ZM11009 & $\begin{array}{l}\text { hpEx4343[Pacr-5-TeTx::RFP + Punc-4-TeTx::RFP + } \\
\text { HygromycinR]; }\end{array}$ & pJH2634; pJH2685 & $\begin{array}{l}\text { eMN chemical } \\
\text { synapse blocked }\end{array}$ \\
\hline ZM7971 & $\begin{array}{l}\text { hpIs321[Pnmr-1-miniSOG::UrSL2::wCherry + lin-15(+)]; } \\
\text { hpIs331[Plgc55-miniSOG::UrSL2::wCherry + lin-15(+)] }\end{array}$ & pJH2827; pJH2890 & eIN ablation \\
\hline ZM11007 & $\begin{array}{l}\text { hpEx4341[Pnmr-1-TexTx::RFP + Plgc55s-TeTx::RFP + } \\
\text { HygromycinR]; }\end{array}$ & pJH4504; pJH2633 & $\begin{array}{l}\text { eIN chemical } \\
\text { synapse blocked }\end{array}$ \\
\hline ZM10206 & $\begin{array}{l}\text { hpEx4080[Prig-3-LoxP::BFP::LoxP::Chrimson::wCherry + } \\
\text { Ptwk-40s-Cre + Pmyo-2-wCherry] }\end{array}$ & pJH4253; pJH4237 & $\begin{array}{l}\text { eIN (AVA) } \\
\text { activation }\end{array}$ \\
\hline ZM7419 & hpIs363[Psra-11-ChR2::YFP + Pttx-3::RFP] ${ }^{67}$ & & $\begin{array}{l}\text { eIN }(\mathrm{AVB}+) \\
\text { activation }\end{array}$ \\
\hline ZM8428 & hpIs459[Punc-4-GCaMP6s::wCherry + lin-15(+)] & pJH3137 & $\mathrm{eMN}(\mathrm{DA}) \mathrm{Ca} 2+$ \\
\hline ZM9128 & hpIs595[Pacr-2s-GCaMP6s::wCherry + lin-15(+)] & pJH3666 & eMN (DB) $\mathrm{Ca} 2+$ \\
\hline ZM7656 & hpIs365[Punc-25-GCaMP3::wCherry + lin-15(+)] & pJH2999 & iMN (DD) $\mathrm{Ca} 2+$ \\
\hline ZM10393 & $\begin{array}{l}\text { hpIs592[Pttr-39-Chrimson::wCherry + lin-15(+)]; hpIs595[Pacr- } \\
\text { 2s-GCaMP6s::wCherry + lin-15(+)] }\end{array}$ & pJH3557; pJH3666 & $\begin{array}{l}\text { eMN Ca2+ upon } \\
\text { iMN activation }\end{array}$ \\
\hline ZM10313 & $\begin{array}{l}\text { hpIs593[Pttr-39-Chrimson::wCherry + lin-15(+)]; } \\
\text { hpIs459[Punc-4-GCaMP6s::wCherry + lin-15(+)] }\end{array}$ & pJH3718; pJH3717 & $\begin{array}{l}\text { eMN (DA) } \\
\text { Ca2+ upon iMN } \\
\text { activation }\end{array}$ \\
\hline ZM10823 & $\begin{array}{l}\text { hpIs596[Pacr-2s-Chrimson::wCherry + lin-15(+)]; } \\
\text { hpIs268[Punc-25-GCaMP3::UrSL2::wCherry + lin-15(+)] }\end{array}$ & pJH3650; pJH2523 & $\begin{array}{l}\text { iMN Ca2+ upon } \\
\text { eMN activation }\end{array}$ \\
\hline ZM10440 & $\begin{array}{l}\text { unc-49(e407) III; hpIs592[Pttr-39-Chrimson::wCherry + lin- } \\
\text { 15(+)]; hpIs595[Pacr-2s-GCaMP6s::wCherry + lin-15(+)] }\end{array}$ & pJH3557; pJH3666 & $\begin{array}{l}\text { eMN Ca2+ upon } \\
\text { iMN activation, } \\
\text { without GABAa }\end{array}$ \\
\hline ZM10673 & $\begin{array}{l}\text { gbb-2(tm1165) IV; hpIs592[Pttr-39-Chrimson::wCherry + lin- } \\
\text { 15(+)]; hpIs595[Pacr-2s-GCaMP6s::wCherry + lin-15(+)] }\end{array}$ & pJH3557; pJH3666 & $\begin{array}{l}\text { eMN Ca2+ upon } \\
\text { iMN activation, } \\
\text { without GABAb }\end{array}$ \\
\hline ZM10585 & $\begin{array}{l}\text { hpEx4177[Pmyo-3::GtACR2::wCherry]; hpIs595[Pacr-2s- } \\
\text { GCaMP6s::wCherry + lin-15(+)] }\end{array}$ & pJH4281; pJH3666 & $\begin{array}{l}\text { eMN Ca2+ upon } \\
\text { muscle inactivation }\end{array}$ \\
\hline ZM9585 & $\begin{array}{l}\text { hpIs615[Pacr-2s-Arch::wCherry + lin-15(+)]; hpIs365[Punc-25- } \\
\text { GCaMP3::wCherry + lin-15(+)] }\end{array}$ & pJH3717; pJH2999 & $\begin{array}{l}\text { iMN Ca2+ upon } \\
\text { eMN inactivation }\end{array}$ \\
\hline ZM7691 & $\begin{array}{l}\text { hpIs371[Punc-4-miniSOG-SL2-wCherry + lin-15(+)]; } \\
\text { hpIs365[Punc-25-GCaMP3::wCherry + lin-15(+)] }\end{array}$ & pJH2843; pJH2999 & $\begin{array}{l}\text { iMN Ca2+ upon } \\
\text { eMN (DA) } \\
\text { ablation }\end{array}$ \\
\hline ZM7798 & $\begin{array}{l}\text { hpIs372[Punc-5-miniSOG-SL2-wCherry + lin-15(+)]; } \\
\text { hpIs365[Punc-25-GCaMP3::wCherry + lin-15(+)] }\end{array}$ & pJH2842; pJH2999 & $\begin{array}{l}\text { iMN Ca2+ upon } \\
\text { eMN (DB) } \\
\text { ablation }\end{array}$ \\
\hline ZM10281 & hpIs740[Ptwk-40s-GCaMP6s::wScarlet] & pJH4260 & $\mathrm{eIN} \mathrm{Ca} 2+$ \\
\hline ZM10341 & $\begin{array}{l}\text { hpIs717[Pacr-2s-LoxP::BFP::loxP::Chrimson::wCherry + Punc- } \\
\text { 17-Cre + lin-15(+)]; hpIs740[Ptwk-40s-GCaMP6s::wScarlet + } \\
\text { lin-15(+)] }\end{array}$ & $\begin{array}{l}\text { pJH4140+pJH4143; } \\
\text { pJH4260 }\end{array}$ & $\begin{array}{l}\text { eIN } \mathrm{Ca} 2+\text { upon } \\
\text { eMN activation }\end{array}$ \\
\hline AQ2953 & ljIs131[Pmyo-3::GCaMP3::SL2::tagRFP-T ] ${ }^{68}$ & & Muscle $\mathrm{Ca} 2+$ \\
\hline ZM10579 & $\begin{array}{l}\text { hpEx4176[Prgef-1-GtACR2::wCherry]; ljIs131 [Pmyo-3- } \\
\text { GCaMP3::UrSL2::tagRFP-T] }\end{array}$ & pJH4329 & $\begin{array}{l}\text { Muscle } \mathrm{Ca} 2+ \\
\text { upon panneural } \\
\text { inactivation }\end{array}$ \\
\hline
\end{tabular}


bioRxiv preprint doi: https://doi.org/10.1101/2021.09.21.461278; this version posted December 21, 2021. The copyright holder for this preprint (which was not certified by peer review) is the author/funder, who has granted bioRxiv a license to display the preprint in perpetuity. It is made available under aCC-BY-NC-ND 4.0 International license.

ZM9648

ZM10575

ZM9429

ZM9573

ZM10464

ZM10339

ZM10340

ZM10458

ZM9131

ZM11020

ZM9172

ZM10451

ZM9313

ZM9551

ZM10176

ZM10441

ZM10410

ZM10411

ZM10743

ZM9119
hpIs673[Prgef-1-Chrimson::UrSL2::wCherry + lin-15(+)]; ljIs 131 [Pmyo-3-GCaMP3::UrSL2::tagRFP-T]

unc-25(e156) III; hpIs673[Prgef-1-Chrimson::UrSL2::wCherry]; 1jIs131 [Pmyo-3-GCaMP3::UrSL2::tagRFP-T]

hpEx4171[Punc-17::GtACR2::wCherry]; ljIs131 [Pmyo3::GCaMP3::UrSL2::tagRFP-T]

zxIs6[Punc-17-ChR2::YFP]; 1jIs131 [Pmyo-3-

GCaMP3::UrSL2::tagRFP-T]

unc-25(e156); zxIs6[Punc-17-ChR2::YFP]; ljIs131 [Pmyo-3GCaMP3::UrSL2::tagRFP-T]

hpEx4138[Prgef-1-HisCl::UrSL2::wCherry]; ljIs131 [Pmyo-3GCaMP3::UrSL2::tagRFP-T] hpIs717[Pacr-2s-LoxP::BFP::LoxP::Chrimson::wCherry + Punc-17-Cre + lin-15(+)]; ljIs131 [Pmyo-3GCaMP3::UrSL2::tagRFP-T] unc-25(e 156) III; hpIs717[Pacr-2sLoxP::BFP::LoxP::Chrimson::wCherry + Punc-17-Cre + lin15(+)]; 1jIs131 [Pmyo-3-GCaMP3::UrSL2::tagRFP-T] hpEx4134[Pacr-2s-LoxP::BFP::LoxP::GtACR2::wCherry + Punc-17-Cre];

ljIs131[Pmyo-3-GCaMP3::UrSL2::tagRFP-T]; hpIs583 [Pacr2s-miniSOG::SL2::RFP+lin-15(+)]

ljIs 131[Pmyo-3-GCaMP3::UrSL2::tagRFP-T]; hpEx4343 [Pacr5-TeTx::RFP + Punc-4-TeTx::RFP + HygromycinR]

unc-25(e156) III; ljIs131 [Pmyo-3-GCaMP3::UrSL2::tagRFP-T]

unc-49(e407) III; ljIs131 [Pmyo-3-GCaMP3::UrSL2::tagRFP-T]

hpIs625[Pttr-39-Arch::wCherry + lin-15(+)]; ljIs131 [Pmyo-3GCaMP3::UrSL2::tagRFP-T]

hpIs593[Pttr-39-Chrimson::wCherry + lin-15(+)]; ljIs131

[Pmyo-3-GCaMP3::UrSL2::tagRFP-T]

unc-25(e156) III; hpIs593[Pttr-39-Chrimson::wCherry + lin-

15(+)]; ljIs131 [Pmyo-3-GCaMP3::UrSL2::tagRFP-T]

unc-49(e407) III; hpIs592[Pttr-39-Chrimson::wCherry + lin-

15(+)]; ljIs 131 [Pmyo-3-GCaMP3::UrSL2::tagRFP-T]

gbb-2(tm1 165) IV; hpIs593[Pttr-39-Chrimson::wCherry + lin15(+)]; ljIs 131 [Pmyo-3-GCaMP3::UrSL2::tagRFP-T]

gbb-1(tm1406) IV; hpIs593[Pttr-39-Chrimson::wCherry + lin15(+)]; ljIs131 [Pmyo-3-GCaMP3::UrSL2::tagRFP-T]

unc-49(e407); gbb-2(tm1165) IV; hpIs592[Pttr-39-

Chrimson::wCherry + lin-15(+)]; ljIs131[Pmyo-3-

GCaMP3::UrSL2::tagRFP-T]

ljIs 131[Pmyo-3-GCaMP3::UrSL2::tagRFP-T]; hpIs559 [Pacr-5miniSOG::SL2::BFP + Punc-4-miniSOG::SL2::BFP + Pttr-39-

miniSOG::SL2::BFP + lin-15(+)]
pJH3628

pJH3628

pJH4330

pJH4407

pJH4140; pJH4143

pJH4140; pJH4143

pJH4211; pJH4143

pJH3626

pJH2634; pJH2685

pJH3718

pJH3557

pJH3557

pJH3557

pJH3557

pJH3557

pJH3557

pJH3439; pJH3449; pJH3458
Muscle Ca2+ upon panneural activation Muscle Ca2+ upon panneural activation, without GABA

Muscle Ca2+ upon pan-cholinergic inactivation Muscle Ca2+ upon pan-cholinergic activation Muscle Ca2+ upon pan-cholinergic activation, without GABA

Muscle $\mathrm{Ca} 2+$ upon panneural silencing Muscle $\mathrm{Ca} 2+$ upon eMN activation

Muscle $\mathrm{Ca} 2+$ upon eMN activation, without GABA Muscle $\mathrm{Ca} 2+$ upon eMN inactivation Muscle $\mathrm{Ca} 2+$ upon eMN ablation Muscle $\mathrm{Ca} 2+$ upon eMN chemical synapse blocked Muscle Ca2+ without GABA Muscle $\mathrm{Ca} 2+$ without GABAa Muscle $\mathrm{Ca} 2+$ upon iMN inactivation Muscle Ca2+ upon iMN activation Muscle $\mathrm{Ca} 2+$ upon iMN activation, without GABA Muscle $\mathrm{Ca} 2+$ upon iMN activation, without GABAa Muscle Ca2+ upon iMN activation, without GABAb Muscle $\mathrm{Ca} 2+$ upon iMN activation, without GABAb Muscle $\mathrm{Ca} 2+$ upon iMN activation, without GABAa/b Muscle $\mathrm{Ca} 2+$ upon all $\mathrm{MN}$ ablation 
bioRxiv preprint doi: https://doi.org/10.1101/2021.09.21.461278; this version posted December 21, 2021. The copyright holder for this preprint (which was not certified by peer review) is the author/funder, who has granted bioRxiv a license to display the preprint in perpetuity. It is made available under aCC-BY-NC-ND 4.0 International license.

ZM7465

hpIs321[Pnmr-1-miniSOG::UrSL2::wCherry + lin-15(+)]; hpIs331[Plgc55-miniSOG::UrSL2::wCherry + lin-15(+)]; ljIs131[Pmyo-3-GCaMP3::UrSL2::tagRFP-T]

ZM10484 unc-25(e156) III; hpIs321[Pnmr-1-miniSOG::UrSL2::wCherry + lin-15(+)]; hpIs331[Plgc55-miniSOG::UrSL2::wCherry + lin-15(+)]; ljIs 131[Pmyo-3-GCaMP3::UrSL2::tagRFP-T]

ZM10311 unc-25(e156); hpEx4080[Prig-3-

LoxP::BFP::LoxP::Chrimson::wCherry + Ptwk-40s-Cre + Pmyo-2-wCherry]; 1jIs131[Pmyo-3::GCaMP3::UrSL2::tagRFPT]

ZM10174 unc-25(e156); hpIs363[Psra-11-ChR2::YFP+ Pttx-3::RFP] ${ }^{23}$; ljIs131[Pmyo-3::GCaMP3::UrSL2::tagRFP-T]

ZM10552

hpEx4081[Prig-3-LoxP::BFP::LoxP::GtACR2::wCherry + Ptwk-40s-Cre + lin-15(+)]; hpIs733[Psra-11-

LoxP::BFP::LoxP::GtACR2::wCherry + Ptwk-40s::Cre + lin-15(+)]; ljIs 131 [Pmyo-3::GCaMP3::UrSL2::tagRFP-T]

ZM11006

EN296

ZM10926

ZM10829 ljIs 131[Pmyo-3-GCaMP3::UrSL2::tagRFP-T]; hpEx4340 [ Pnmr-1-TeTx::RFP + Plgc-55s-TeTx::RFP+HygromycinR ]

unc-49(kr296::tagRFP $)^{28}$

$h p I s 827$ [ GBB-1::GFP (fosmid) + Pmyo-2::RFP ]

$h p E x 4271$ [ GBB-2::GFP (fosmid) + Pmyo-2::RFP ]

\begin{tabular}{l|l} 
pJH2827; pJH2890 & $\begin{array}{l}\text { Muscle Ca2+ upon } \\
\text { eIN ablation }\end{array}$ \\
pJH2827; pJH2890 & $\begin{array}{l}\text { muscle Ca2+ } \\
\text { upon eIN ablation, } \\
\text { without GABA } \\
\text { Muscle Ca2+ } \\
\text { upon eIN (AVA) } \\
\text { pJH4253;pJH4237 } \\
\text { activation, without } \\
\text { GABA } \\
\text { Muscle Ca2+ } \\
\text { upon eIN (AVB) } \\
\text { activation, without } \\
\text { GABA } \\
\text { Muscle Ca2+ upon } \\
\text { eIN inactivation } \\
\text { pJH4217; } \\
\text { pJH4235; pJH4237 } \\
\text { pJH4505; pJH2633 } \\
\text { pJH4578 }\end{array} \mid \begin{array}{l}\text { Muscle Ca2+ } \\
\text { upon eIN chemical } \\
\text { synapse blocked } \\
\text { GABAa expression } \\
\text { GABAb expression } \\
\text { GABAb expression }\end{array}$ \\
\hline
\end{tabular}

